\title{
OPEN Uncovering the hidden bacterial ghost communities of yeast and experimental evidences demonstrates yeast as thriving hub for bacteria
}

\author{
B. Indu ${ }^{1,3}$, Tallapragada Keertana ${ }^{1,3}$, Sahu Ipsita ${ }^{1}$, Uppada Jagadeeshwari ${ }^{2}$, \\ Chintalapati Sasikala ${ }^{2} \&$ Chintalapati Venkata Ramana ${ }^{1 凶}$
}

Our major concern was to address "yeast endobacteria" which was based on a few reports in the recent past where bacteria may find yeast as a niche for survival. In this study, we report the microbiota of twenty-nine axenic yeast cultures recovered from different habitats based on their $16 \mathrm{~S}$ rRNA gene-amplicon metagenomes. Yeasts were identified based on D1/D2 or ITS gene sequences. Bacterial diversity was widespread, varied and rich among all yeasts except for four strains. Taxa belonging to the phylum Firmicutes, Proteobacteria, Actinobacteria and Bacteroidetes and the genera; Streptococcus, Propionibacterium were common to all the yeasts. Candida tropicalis was used as a model organism to confirm bacteria through fluorescence in situ hybridization (FISH), isolating and re-introducing the isolated bacteria into the yeast. FISH analysis confirmed the endobacteria of C. tropicalis and we have successfully isolated four bacteria only after lysis and disruption of yeast cells. These bacteria were identified as species of Pseudomonas, Chryseobacterium, Lysinibacillus and Propionibacterium. Guestimates indicate $95 \%$ of bacterial species of $C$. tropicalis are yet-to-becultivated. We have successfully reintroduced $m$ Cherry tagged Pseudomonas into $C$. tropicalis. Also, auto-fluorescent Prochlorococcus and Rhodopseudomonas could be introduced into $C$. tropicalis while $\mathrm{mCherry}$ tagged $E$. coli or Salmonella could not be introduced. FISH analysis confirmed the presence of both native and infected bacterial cells present in $C$. tropicalis. Our findings unveil the insights into the ghost microbiota associated with yeast, which otherwise are considered to be axenic cultures. Their inherent occurrence, together with co-cultivation experiments under laboratory conditions suggests that yeasts are a thriving hub for bacterial communities.

There is a saying among microbial ecologists that no organism lives in isolation. The importance of interactions and relationships between different organisms, even if it is a unicellular one cannot be ignored ${ }^{1}$. Of these, bacteria-fungus interactions (BFI) have been only recently investigated in detail, giving us a different perspective into the nature and impacts of these relationships ${ }^{2,3}$. They are very important because fungi and bacteria are commonly found together in many natural communities ${ }^{2-7}$. Studies on BFI have shown the role of both organisms in influencing each other's biology as well as their influence on the ecosystem ${ }^{1-5}$. For instance, the bacterial endosymbionts of soil fungus Rhizopus control the molecular reins of the fungus to become heritably transmitted ${ }^{6}$. Other interesting cases include the recently uncovered relationships between plant-associated filamentous fungi and their endobacteria, some of which were shown to be uncultivable ${ }^{2,3,7}$. Such interesting cases show the newly uncovered facet of microbial interactions, where dynamics previously thought to exist between certain partners (for example plant-fungus) have now been extended to the bacterial endosymbionts (for example, plant-fungus-endobacteria). However, bacterial interactions (particularly endobacteria) with yeast, remains obscure and not much is still known.

\footnotetext{
${ }^{1}$ Department of Plant Sciences, School of Life Sciences, University of Hyderabad, Hyderabad, India. ${ }^{2}$ Bacterial Discovery Laboratory, Centre for Environment, Institute of Science and Technology, J.N.T. University Hyderabad, Hyderabad, India. ${ }^{3}$ These authors contributed equally: B. Indu and Tallapragada Keertana. ${ }^{\circledR}$ email: cvr449@ gmail.com
} 
Yeasts engage in a wide variety of relationships ${ }^{8}$ such as predation ${ }^{9}$ and processes like autophagy ${ }^{10}$. Documented shreds of evidence show that many times, yeasts and bacteria co-exist in the same environment ${ }^{11}$ and yet, their interactions have not been studied in depth. Fungal microbiomes are becoming a thriving area of research, showing how distinct each microbiome is to the fungal host ${ }^{2,3,7}$. It has been speculated that such fungal microbiomes may contribute to the host biology just like the animal and plant microbiomes $\mathrm{do}^{2}$. Their roles are different in different fungi. For example, Burkholderia rhizoxinica has a positive effect on Rhizopus whereas Candidatus Moeniiplasma glomeromycotorum (CaMg) has a negative impact on Mucoromycota ${ }^{3}$. Well-studied cases of fungal endobacteria to date have been mostly restricted to few fungal hosts like the AMF and other plant-associated filamentous fungi ${ }^{2-4,6}$. Although some research related to bacterial 'endosymbionts' of yeasts is still underway ${ }^{12-17}$, such cases are sparse in number and can be counted on the fingers. One of the earliest reports among these was of a possible symbiotic relationship between Candida tropicalis and Microbacterium sp. ${ }^{17}$. Other reports demonstrated Helicobacter cells inside yeast vacuoles by immunofluorescence, where vacuoles were considered as a specialized niche for such bacteria ${ }^{12,14}$. Localization of Staphylococcus in yeast vacuole was also similarly shown ${ }^{15}$. Work on Saccharomyces and the endosymbiont Wolbachia, demonstrated the latter's infection in the yeast, allowing for the study of host-endosymbiont dynamics ${ }^{16}$. Yeast-bacterial interactions, particularly the endobacteria phenomenon was speculated as potentially important to nectar research, where both these microorganisms play a key role in the ecology and biochemistry of flower nectar ${ }^{5}$. Similarly, the yeast Rhodotorula mucilaginosa was shown to harbor endosymbiotic Pseudomonas stutzeri ${ }^{18}$. This yeast-endobacteria system has been implicated in interactions with rice plant that hosts the yeast, wherein these microorganisms collectively aid in nitrogen fixation ${ }^{19}$. Additionally, there has been growing interest to artificially generate yeast-endobacteria systems to understand the endosymbiosis theory ${ }^{20,21}$. Such simulations aim to fuel further research on endosymbiosis and to allow the elucidation of events involved. We explored the fascinating occurrence of bacteria associated with yeasts, which started with our serendipitous observation of moving Bacteria-Like-bodies (BLBs) inside yeast cells. We begin by presenting the diversity of yeasts and their bacteria. We also made attempts to isolate some of these bacteria and infect them to the yeast, in order to similarly relate to Koch's postulates.

\section{Methods}

Isolation, identification and preservation of yeast. Environmental samples collected from different geographical regions of India were used as sources for yeast isolation which include soils, fruits and other biological sources (Table 1). Samples were inoculated in Yeast Peptone Dextrose (YPD) broth (HIMEDIA, M1363) containing chloramphenicol $\left(100 \mu \mathrm{g} \mathrm{mL}^{-1}\right)$ and kept for enrichment for $48-72 \mathrm{~h}$ at $30^{\circ} \mathrm{C}$. Enrichment cultures were streaked onto YPD agar with chloramphenicol and incubated at $30^{\circ} \mathrm{C}$ for $4-5$ days. Isolated colonies were repeatedly streaked to obtain axenic cultures which were preserved as glycerol stocks $(50 \% \mathrm{v} / \mathrm{v})$ at $-20^{\circ} \mathrm{C}$. Purity of the yeast cultures was confirmed by streaking the yeast on nutrient agar and simultaneously observing under microscope. A total of 29 yeast strains were isolated, purified and preserved. They were named in the JY series starting from JY101.

Isolation of genomic DNA, PCR amplification and identification of yeast. Genomic DNA of all the yeast strains was isolated using the method described by Hoffman ${ }^{22}$. Briefly, cells were lysed with Triton-X 100 and glass beads. DNA was extracted using phenol-chloroform-isoamyl alcohol and eluted with Tris EDTA buffer. For the identification of yeast, internal transcribed spacer (ITS) region was amplified with ITS1 (5'-GTC GTAACAAGGTTTCCGTAGGTG-3') and ITS4 (5'-TCCTCCGCTTATTGATATGC-3') primers and D1/D2 domain of 26S rRNA gene was amplified using NL1 (5'-GCATATCAATAAGCGGAGGAAAAG-3') and NL4 (5'-GGTCCGTGTTTCAAGACGG-3') primers respectively. DNA sequencing of the amplified regions were outsourced to AgriGenome Labs Pvt Ltd, India. BLAST analysis in the NCBI database was carried out for the sequences and identification of yeast was performed using the YeastIP database and CBS database $\mathrm{s}^{23}$ (http://www. westerdijkinstitute.nl). All the sequences were submitted in the NCBI database and the accession numbers are given in Table 1.

Staining and microscopy. Yeast cells were observed under phase contrast (Leica DFC295) microscopy where moving bacteria like bodies (BLBs) in the yeast cell were seen. To stain and observe these BLBs, ViaGram (Invitrogen, V7023) kit containing DAPI, SYTOX and Texas Red conjugated with wheat germ agglutinin (WGA) was used. Yeast cells were washed thrice with BSA- $0.9 \% \mathrm{NaCl}$ buffer and then incubated first with Texas Red for 15 min. Excess stain was removed by washing the incubated cells with BSA- $0.9 \% \mathrm{NaCl}$ buffer. To the washed cells, $2 \mu \mathrm{L}$ mixture of DAPI and SYTOX (prepared according to the kit instructions) was added and incubated for $15 \mathrm{~min}$ in the dark. Cells were then observed under confocal microscope (Zeiss LSM880) which showed fluorescent red-stained BLBs under the excitation/emission maxima set to $\sim 595 / 615 \mathrm{~nm}$.

Purity of yeast cultures, 16S rRNA gene metagenome analysis of V1-V3 region. The following protocols were strictly followed to ensure absence of extracellular bacteria before DNA extraction. Yeast strains were cultured on various bacterial media [Luria broth (HIMEDIA, M575), Thiobacillus agar (HIMEDIA, M788), Mueller Hinton agar (HIMEDIA, M173)] to confirm bacterial contamination. Contamination was also checked by observing yeast cells under phase contrast microscope and confocal microscope after staining the cells with Texas Red. In addition, the purity of yeast cultures was further confirmed by repeated subculturing in YPD media containing chloramphenicol $\left(100 \mu \mathrm{g} \mathrm{mL}^{-1}\right)$. DNA was extracted from the yeast cells by the method described by Hoffman ${ }^{22}$, from equivalent biomass of all yeast strains grown in YPD broth harvested at OD of 0.8 . Amplification and sequencing of the V1-V3 region of the 16S rRNA gene were outsourced to RTL Genomics, USA and Eurofins Scientific, India, which was performed on Illumina MiSeq platform. As a control, samples 


\begin{tabular}{|c|c|c|c|c|}
\hline Source & Strain & Similarity with the nearest yeast & D1/D2 or ITS accession & $\begin{array}{l}\text { Metagenome accession } \\
\text { number of the yeast }\end{array}$ \\
\hline Soil & JY101 & Candida tropicalis CBS $94^{\mathrm{T}}$ & LT999794 & SRR7746671 \\
\hline Date fruit & JY106 & ${ }^{*}$ Candida tropicalis $\mathrm{CBS} 94^{\mathrm{T}}$ & LT719072 & SRR7752355 \\
\hline Lichen & JY107 & ${ }^{*}$ Candida tropicalis $\mathrm{CBS} 94^{\mathrm{T}}$ & LT719073 & SRR7752303 \\
\hline Leaf litter & JY108 & ${ }^{*}$ Candida tropicalis CBS $94{ }^{\mathrm{T}}$ & LT719074 & SRR7752354 \\
\hline Citrus fruit & JY113 & ${ }^{*}$ Candida tropicalis $\mathrm{CBS} 94^{\mathrm{T}}$ & LT795049 & SRR7757783 \\
\hline Decomposed wood & JY114 & ${ }^{\star}$ Candida tropicalis $\mathrm{CBS} 94^{\mathrm{T}}$ & LT795050 & SRR7757789 \\
\hline Biofilm & JY125 & Candida tropicalis ATCC $750^{\mathrm{T}}$ & LT838873 & SRR7762712 \\
\hline Carve soil & JY134 & Candida tropicalis CBS $94^{\mathrm{T}}$ & LT995997 & SRR7774106 \\
\hline Sewage water & JY103 & $\begin{array}{l}{ }^{*} \text { Candida metapsilosis CBS } \\
10907^{\mathrm{T}}\end{array}$ & LT719069 & SRR7752356 \\
\hline Muskmelon & JY110 & ${ }^{*}$ Candida intermedia $\mathrm{CBS} 572^{\mathrm{T}}$ & LT795046 & SRR7757784 \\
\hline Brown algae Gracillaria sp. & JY121 & Candida suratensis CBS $10928^{\mathrm{T}}$ & LS992563 & SRR7760802 \\
\hline Brown algae Gracillaria sp. & JY124 & Candida suratensis CBS $10928^{\mathrm{T}}$ & LT840079 & SRR7762711 \\
\hline Kadam fruit & JY135 & Candida ampae CBS 7872 & LT996821 & - \\
\hline Gooseberry & JY105 & ${ }^{*}$ Pichia kudriavzevii CBS $5147^{\mathrm{T}}$ & LT719071 & SRR7752353 \\
\hline Idly batter & JY112 & ${ }^{\star}$ Pichia kudriavzevii CBS $5147^{\mathrm{T}}$ & LT795048 & SRR7757792 \\
\hline \begin{tabular}{|l|} 
Sugarcane \\
\end{tabular} & JY116 & ${ }^{*}$ Pichia kudriavzevii CBS $5147^{\mathrm{T}}$ & LT795052 & SRR7757791 \\
\hline Lichen & JY131 & $\begin{array}{l}\text { Pichia kudriavzevii NRRL } \\
\text { Y-5396 }^{\mathrm{T}}\end{array}$ & LT962958 & SRR7774038 \\
\hline Mushroom & JY129 & $\begin{array}{l}\text { Pichia kudriavzevii NRRL } \\
\text { Y-5396 }^{\mathrm{T}}\end{array}$ & LT962960 & SRR7762710 \\
\hline Fruit Neolamarckia cadamba & JY136 & Pichia kluyveri NRRL y-11519 ${ }^{\mathrm{T}}$ & LT996820 & SRR7774105 \\
\hline Idly batter & JY104 & $\begin{array}{l}{ }^{*} \text { Meyerozyma guilliermodii CBS } \\
2030^{\mathrm{T}}\end{array}$ & LT719070 & SRR7752352 \\
\hline Citrus fruit & JY117 & $\begin{array}{l}{ }^{*} \text { Meyerozyma carribicca } \text { CBS } \\
9966^{\mathrm{T}}\end{array}$ & LT795053 & SRR7757782 \\
\hline Grapes & JY102 & $\begin{array}{l}\text { Hanseniaspora guilliermondii } \\
\text { CBS } 465^{\mathrm{T}}\end{array}$ & LT999810 & SRR7752479 \\
\hline Soil & JY109 & $\begin{array}{l}{ }^{*} \text { Rhodotorula mucilaginosa CBS } \\
316^{\mathrm{T}}\end{array}$ & LT795045 & SRR7752357 \\
\hline Algal growth & JY127 & $\begin{array}{l}\text { Rhodotorula mucilaginosa CBS } \\
316\end{array}$ & LT799394 & - \\
\hline Mushroom & JY132 & $\begin{array}{l}\text { Rhodotorula mucilaginosa CBS } \\
316\end{array}$ & LT962959 & - \\
\hline Lake sediment & JY143 & $\begin{array}{l}\text { Rhodotorula mucilaginosa CBS } \\
316\end{array}$ & LT996827 & - \\
\hline Pine tree bark & JY119 & Zalaria obscura DAOMC $250849^{\mathrm{T}}$ & LT840078 & SRR7757780 \\
\hline Mushroom & JY130 & $\begin{array}{l}\text { Debaryomyces prosopidis JCM } \\
9913^{\mathrm{T}}\end{array}$ & LT962955 & SRR7762713 \\
\hline Soil & JY133 & \begin{tabular}{|l|} 
Sporidiobolus pararoseus CBS \\
$491^{\mathrm{T}}$
\end{tabular} & LT962957 & \\
\hline
\end{tabular}

Table 1. Source, highest identity and accession numbers of D1/D2, ITS or metagenome of yeasts used in this study. -, not yielded results. ${ }^{\star}$ Indicates the identification based on ITS sequencing.

without yeast biomass were also processed for DNA extraction protocol and sequencing. The analysis and taxonomy assignment to the sequences were done using mothur software package, version $1.41 .1^{24}$. The protocol described by Schloss et al. for analysis of MiSeq data was followed ${ }^{25}$. First, the paired-end reads were assembled into contigs and screened for quality and removal of chimeras. The minimum length of sequences was restricted to $350 \mathrm{bp}$ and the maximum length was limited to $550 \mathrm{bp}$ to correspond to the size of the V1-V3 region of the 16S rRNA gene. The screened sequences were aligned to the SILVA 16S rRNA gene sequence database ${ }^{26}$. Sequences were again evaluated for chimeras, ambiguous, homopolymers and undesirable sequences such as those belonging to organelles, Archaea, eukaryotes were removed.

Aligned sequences were clustered into operational taxonomic units (OTUs) using 0.03 divergence cut-off. The raw sequences were submitted in the NCBI database and the accession numbers are given in Table 1 against each yeast strain. Diversity and comparison of the data along with graphical representation was done using Microsoft Excel and web-based tools. The MicrobiomeAnalyst online tool ${ }^{27}$ was used to filter data for the low count and low variance and normalize it by total sum-scaling for alpha and beta diversity measurements. Data was also rarefied as recommended to minimum library size. The Chao-1 index was used to measure alpha diversity and species richness with the statistical method set to T-test/ANOVA for significance testing. Bray-Curtis index calculated for beta diversity assessment among the samples was visualized as Principal Coordinates Analysis (PCoA) plots. Permutational multivariate analysis of variance was used to test the significance of the index. The common and 
unique features (phyla and genera) were evaluated visually in the form of Venn diagrams constructed using the InteractiVenn web-based tool ${ }^{28}$. Heatmaps were created using the Heatmapper online tool ${ }^{29}$ with the normalized data. Row and column clustering using average linkage was applied by Euclidean measurement of the distances. PAST V3.26 software was used to construct the rarefaction curves ${ }^{30}$.

Isolation and cultivation of bacteria associated with yeast. As the metagenome results revealed the presence of more than one type of bacteria with the yeast cell, we used several approaches to isolate these bacteria from axenic yeast culture. These included zymolase treatment which degrades the cell wall of yeast and shearing of yeast cells via vortexing with glass beads. In the latter method, Candida tropicalis was grown in nutrient broth containing $100 \mu \mathrm{g} \mathrm{mL} \mathrm{m}^{-1}$ of chloramphenicol for about $98 \mathrm{~h}$. Cells were centrifuged and sterile glass beads were added. This was vortexed vigorously to shear the yeast cells. The sheared yeast cells were centrifuged at $6000 \times \mathrm{g}$ for 5 min for settling down the yeast cell debris. Being lighter in weight, bacterial cells were presumed to remain in the supernatant. Hence, the supernatant was serially diluted up to $10^{-5}$ dilution and plated onto a nutrient agar plate containing cycloheximide $\left(200 \mu \mathrm{g} \mathrm{mL}^{-1}\right)$. Plates were then incubated at $30^{\circ} \mathrm{C}$ for 1 week. Cultures with yellow and white color colonies were seen on the plates which were further purified by repeated sub-culturing. DNA of the purified colonies was extracted and the $16 \mathrm{~S}$ rRNA gene for each isolate was amplified using F27 and R1525 primers as described earlier ${ }^{31}$. The amplified product was sent for sequencing to Agrigenome Labs Pvt Ltd, India. The sequenced product was searched with BLAST in EzBiocloud to get the identity of the nearest taxa.

Fluorescence in situ hybridization. Fluorescence in situ hybridisation (FISH) is a powerful tool to enumerate and specify the bacteria in environmental samples or mixed cultures ${ }^{32}$. This protocol uses the oligonucleotide probe targeting the $16 \mathrm{~S}$ rRNA of bacteria. Briefly, $2 \mathrm{~mL}$ of overnight grown yeast cells were centrifuged at 13,000 rpm for $5 \mathrm{~min}$. Supernatant was discarded and the cells were fixed in $1 \mathrm{~mL}$ of fixative solution (4\% formaldehyde in PBS buffer). Fixed cells were incubated for $3 \mathrm{~h}$ at room temperature and then centrifuged at 13,000 rpm for $5 \mathrm{~min}$. The pellet obtained was resuspended in $50 \%$ ethanol and incubated for $5 \mathrm{~min}$ at room temperature. Resuspended pellet was centrifuged at 13,000 rpm for $5 \mathrm{~min}$. Pellet was again washed with $80 \%, 95 \%$ ethanol and air dried the cell by placing it in speed vacuum for $10 \mathrm{~min} .500 \mu \mathrm{L}$ of hybridisation buffer (Solution of $20 \mathrm{mM}$ TrisCl, $0.9 \mathrm{M} \mathrm{NaCl}, 0.01 \%$ SDS in $40 \%$ formamide) was added to dried cells and incubated at $37^{\circ} \mathrm{C}$ for $30 \mathrm{~min}$ thereby firming pre-hybridisation mixture. $10 \mu \mathrm{L}$ of bacterial specific probe EUB338-Cy3 $\left(10 \mathrm{pmol} \mu \mathrm{L}^{-1}\right.$ of working stock with sulfoindocyanine dye Cy3) was added to $50 \mu \mathrm{L}$ of the pre-hybridisation mixture and incubated for $24 \mathrm{~h}$ at $50^{\circ} \mathrm{C}$. After $24 \mathrm{~h}$, cells were centrifuged at $13,000 \mathrm{rpm}$ for $5 \mathrm{~min}$ and washed thrice in $0.1 \mathrm{X}$ SSC buffer. The final pellet was dissolved in $20 \mu \mathrm{L}$ of $0.1 \mathrm{X}$ SSC buffer. Slides were prepared and observed under confocal microscope with emission wavelength at $499-542 \mathrm{~nm}$ and excitation at $543 \mathrm{~nm}$.

Fluorescence labelling of Pseudomonas stutzeri JC703, Escherichia coli DH5 $\alpha$ and Salmonella typhimurium. Pseudomonas stutzeri JC703, Escherichia coli DH5 $\alpha$ and Salmonella typhimurium were tagged with fluorescent protein mCherry contained in a plasmid carrying antibiotic cassette for its maintenance in bacteria. Here, pBBRMCS-5 was used as the cloning vector which has a broad host range for Gram-negative bacteria carrying a gentamycin resistant cassette. The mCherry gene was cloned into pBBRMCS-5 plasmid under the constitutive expression of tac promoter as described earlier ${ }^{33}$. The constructed plasmid pBBRMCS- 5 harboring the mCherry fluorescent tag and gentamycin resistant gene cassette was then transformed in the P. stutzeri JC703, E. coli DH5a and S. typhimurium.

Transformation of P. stutzeri JC703 was done using the heat shock protocol described by Feng and coworkers $^{34}$, with a few modifications. Briefly, $100 \mu \mathrm{L}$ of overnight grown P. stutzeri JC703 was inoculated in $10 \mathrm{~mL}$ of Luria broth (HIMEDIA) and incubated at $30^{\circ} \mathrm{C}$ for $2-3 \mathrm{~h}$ until the OD reached $0.4-0.5$. The culture was then kept on ice for $30 \mathrm{~min}$ followed by centrifuging $2 \mathrm{~mL}$ of culture at $7000 \times \mathrm{g}$ for $10 \mathrm{~min}$. The pellet was washed three times in water and dissolved in $1 \mathrm{~mL}$ of $0.1 \mathrm{M} \mathrm{CaCl}_{2} \cdot 2 \mathrm{H}_{2} \mathrm{O}$ (Sigma). Cells were incubated on ice for $1 \mathrm{~h}$ and centrifuged at $7000 \times \mathrm{g}$ for $10 \mathrm{~min}$. The pellet was dissolved in $1 \mathrm{~mL}$ of a solution containing $0.7 \mathrm{~mL}$ of $\mathrm{CaCl}_{2} \cdot 2 \mathrm{H}_{2} \mathrm{O}$ and $0.3 \mathrm{~mL}$ of $50 \%$ glycerol. The above steps were performed to make the cells competent to receive foreign DNA. The competent Pseudomonas cells were then used for transformation with the pBBRMCS plasmid. Competent $P$. stutzeri JC703 cells were incubated with the plasmid on ice for $30 \mathrm{~min}$ followed by heat shock at $42{ }^{\circ} \mathrm{C}$ for $6 \mathrm{~min}$. Cells were immediately transferred to the ice for $3 \mathrm{~min}$ and then incubated in $0.9 \mathrm{~mL}$ of Luria broth (HIMEDIA) at $30^{\circ} \mathrm{C}$ for $2 \mathrm{~h}$. Cells were centrifuged and $0.9 \mathrm{ml}$ of the supernatant was discarded and the remaining was spread onto LB agar plates containing gentamycin $\left(25 \mu \mathrm{g} \mathrm{m} \mathrm{L}^{-1}\right)$. The plates were incubated at $30^{\circ} \mathrm{C}$ for $48 \mathrm{~h}$ and screened for the presence of transformed colonies. The same protocol was followed for the transformation of E. coli DH5a and S. typhimurium with a few modifications, where heat shock was given for $90 \mathrm{~s}$ followed by incubation at $37^{\circ} \mathrm{C}$ for an hour. After incubation, cells were centrifuged and pellets were dissolved in $100 \mu \mathrm{L}$ of Luria broth (HIMEDIA, M575). The dissolved pellets were spread onto LB agar (HIMEDIA, $\mathrm{M} 557+$ agar) plates containing gentamycin $\left(25 \mu \mathrm{g} \mathrm{mL}{ }^{-1}\right)$. The plates were incubated at $37^{\circ} \mathrm{C}$ overnight and screened for the presence of transformed colonies.

Infection of yeast with fluorescent bacteria. The transformed fluorescent $P$. stutzeri JC703, E. coli $\mathrm{DH} 5 \mathrm{a}$ and S. typhimurium as well as auto-fluorescent Prochlorococcus sp. and Rhodopseudomonas palustris were used for infecting C. tropicalis JY101. Cells were first grown in Yeast Carbon Base (YCB) broth (HIMEDIA, $\mathrm{M} 141$ ) in the absence of additional nitrogen sources at room temperature for $24 \mathrm{~h}$. The fluorescently labelled P. stutzeri JC703, E. coli DH5a, S. typhimurium or the auto-fluorescent Rhodopseudomonas palustris TIE-1 and Prochlorococcus sp. cells were co-cultured with C. tropicalis cells in YCB medium devoid of additional nitrogen sources at room temperature. Co-cultures were observed periodically under confocal microscope (Zeiss 
LSM880) with 63X objective lens. Cells were processed for scanning electron microscopy as described earlier ${ }^{35}$ and the images were taken on Philips XL3O SEM. The co-cultures of $C$. tropicalis and mCherry labelled fluorescent $P$. stutzeri were also analysed by FISH as described above and observed under confocal microscope.

\section{Results}

Diversity and identity of yeasts. A total of twenty-eight yeasts and one yeast-like fungus were isolated and purified from diverse habitats (Table 1). These were identified based on the D1/D2 domain of large ribosomal subunit or ITS regions (Table 1). Eight strains (JY101, JY106, JY107, JY108, JY113, JY114, JY125, JY134) had the highest (>99\%) identity with Candida tropicalis. While other species of the genus Candida include; Candida metapsilosis (JY103), Candida suratensis (JY121, JY124) and Candida ampae (JY135). Six strains (JY105, JY112, JY116, JY131, JY129, JY136) showed maximum identity (99\%) with the genus Pichia. Four strains belonged to the genus Rhodotorula (JY109, JY127, JY132, JY143), two strains (JY104 and JY117) to Meyerozyma, and a single strain each belonging to the genera Hanseniaspora (JY102), Debaryomyces (JY130) and Sporidiobolus (JY133) (Table 1). The yeast-like fungus with strain number JY119 was identified as Zalaria obscura. All the strains were preserved in $50 \%$ glycerol at $-20^{\circ} \mathrm{C}$.

Confirmation of axenic cultures of yeast. As described in methodology, multiple methods were adopted to establish the "axenic" nature of all yeast cultures. This was mainly done by screening all yeast strains using: repeated subculturing on various media, microscopic studies of yeast cells with viability stains, FISH analysis and microscopy. These methods demonstrated no external contamination of bacteria.

Microscopic observation of bacteria like bodies. While observing the morphology of yeast cells under phase-contrast microscope, rapidly moving bodies roughly the size of bacteria were seen in yeast cells (Fig. S1a and Movie S1). Simple bacterial fluorescent staining was done using Texas Red (Fig. S1b) and also the yeast DNA was counterstained with DAPI/SYTOX (Fig. S1c; Movie S2). Twenty-five out of twenty-nine strains of yeast showed such bacteria like bodies (BLBs) in their cells as confirmed from staining. Further, we also observed more than one BLB in the yeast (Movie S2) for which we looked into the bacterial diversity of yeast through metagenome sequencing. FISH was also performed for $C$. tropicalis using rRNA bacteria specific probe EUB338Cy3.

Composition and diversity of bacterial communities. Before the extraction of the genomic DNA of yeast, we made sure that there were no external bacteria as observed under the confocal microscope after staining the bacteria. Genomic DNA was extracted from all the twenty-nine strains of yeast and 16S rRNA genebased metagenome sequencing of the V1-V3 region was performed. Except for the yeast strains JY127, JY132, JY135 and JY143, the remaining twenty-four yeast strains as well as yeast-like fungal strain JY119 yielded good sampling depth as observed from the rarefaction curves (Fig. S2). The same four strains also yielded negative results for staining, suggesting that they may lack bacteria. The metagenome data of the yeasts are deposited with NCBI and the Sequence Read Archive (SRA) accession numbers are given in Table 1. Since Candida and Pichia were among the largest (represented by about $48 \%$ and $24 \%$, respectively) of our collection of yeast in this study, we present the metagenome results of these separately.

Bacterial community of Candida spp. The highest number of OTUs (3181) was observed from strain JY121 and lowest number (529) from JY101. Firmicutes constituting approximately $49 \%$ of the OTUs were the most abundant phylum in all strains of Candida (Figs. 1a, 2a, 3Ia). Other reads mapping to Actinobacteria $(\sim 20 \%)$, Bacteroidetes ( 2\%), Proteobacteria $(\sim 20 \%)$ and unclassified bacteria $(\sim 7 \%)$ were common to the four species (Fig. 1a). Further, differences in their bacterial composition are presented in the beta diversity plot using Bray-Curtis index with the permutational MANOVA (PERMANOVA) statistical method. The plot shows (Fig. 3Ia) the individuality of OTUs make-up in each strain, clearly displaying no correlation among them, despite sharing some common trends at the level of phylum (Fig. 3Ia) or genus (Fig. 3IIa). Further comparison at the phylum level among these strains is given in Table S1 and Venn diagram (Fig. 3Ib).

Varied results were observed at the genus level where Streptococcus $(\sim 10 \%)$ was common among all the Candida spp. with maximum OTUs (210) in strain JY103. Planococcus reads were highest in strain JY121 with 446 OTUs. Genera like Corynebacterium ( 2\%), Propionibacterium ( 3\%), Staphylococcus ( 7\%), unclassified bacteria ( $\sim 9 \%)$ and unclassified Actinomycetales ( 6\%) were also common to all Candida spp. (Figs. 1b, 2b, 3IIb). Besides, unclassified Pseudomonadaceae reads were unique to strain JY101 having 25 OTUs (Table S2; Fig. 3IIb).

We further compared six strains of C. tropicalis (JY101, JY107, JY108, JY113, JY114 and JY125) for intraspecies analysis of bacterial diversity. Yeast strain JY125 had the greatest number of OTUs (3125) and strain JY101 had the least (529). Deinococcus-Thermus, Spirochaetes, Gemmatimonadetes were the unique phyla of bacterial diversity of C. tropicalis strains JY101, JY107 and JY114 respectively (Fig. S3Ia,Ib; Table S3). Firmicutes constituted approximately half the total read share (up to 46\%) in these strains. Actinobacteria $(\sim 22 \%)$ and Proteobacteria $(\sim 23 \%)$ were the next abundant phyla with a similar number of reads. Genus level analysis (Fig. S3IIa,IIb; Table S4) showed the presence of the genus Staphylococcus, unclassified bacteria and unclassified Actinomycetales in all the C. tropicalis strains. Although diversity was more varied at the genus-level, few taxa showed high read dominance. For example, Staphylococcus ( 54\%) was abundant in strain JY114 while Streptococcus and Planococcus reads were in similar proportions in the rest of the C. tropicalis strains. As mentioned previously, unclassified Pseudomonadaceae reads only showed up in strain JY101. 

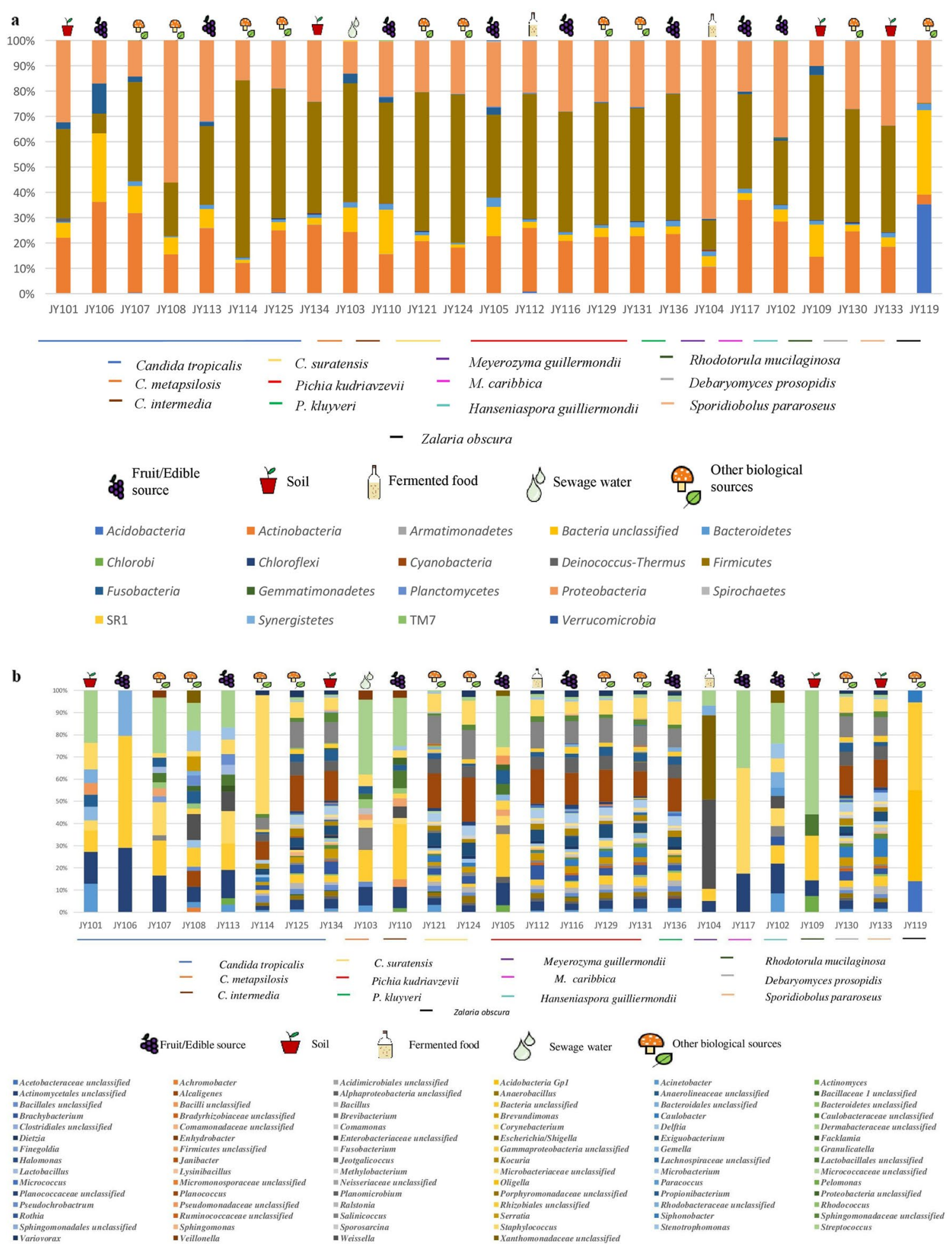

Figure 1. Stacked bar plots showing the relative abundances of bacterial taxa reads distributed among the twenty-five yeast strains; (a), taxa at phylum level; (b), bacteria at genus level. X-axis indicates yeast strains and $\mathrm{Y}$-axis indicates the relative abundance of bacterial taxa calculated as percentage of Operational Taxonomic Units (OTUs). The plots are supported with legends showing the yeast strains, bacterial taxa as well as environmental sources of yeast isolation. Data on bacterial taxa reads found in each yeast was obtained using mothur V. 1.41.1 $1^{24-26}$. Plots were constructed using Microsoft Excel and images are self-made using Microsoft power point. 
a
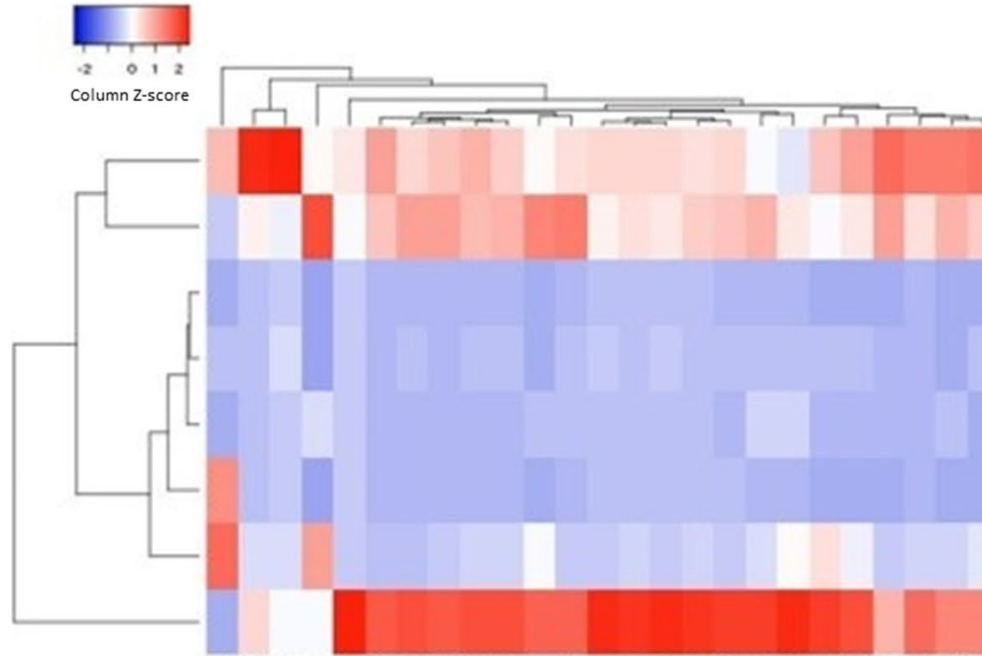

Proteobacteria

Actinobacteria

Chloroflexi

Bacteroidetes

Fusobacteria

Acidobacteria

Unclassified bacteria

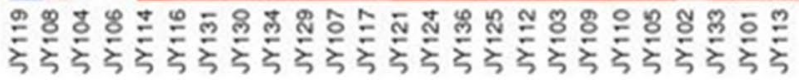

\section{Firmicutes}

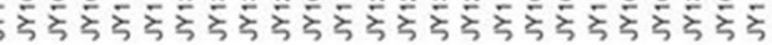

b

b

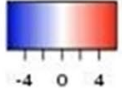
$\begin{array}{ccc}-4 & 0 & 4 \\ \text { Column Z-Scare }\end{array}$
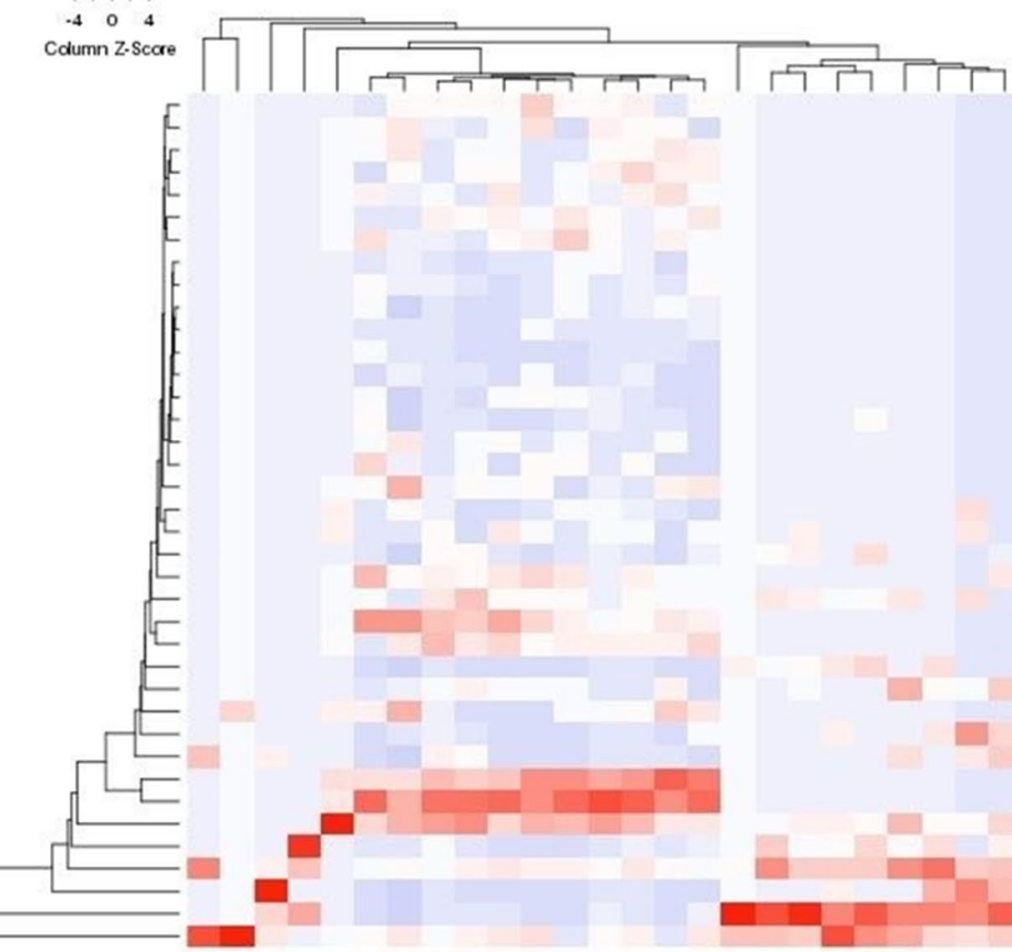

Unclassified Sphingomonadaceae Anaerobacillus Variovorax Unclassified Rhizobiales Kocuria

Microbacterium

Brevundimonas

Unclassified Planococcaceae

Unclassified Microbacteriaceae

Micrococcus

Unclassified Bradyrhizobiaceae

Comamonas

Unclassified Comamonadaceae

Dietzia

Unclassified Alphaproteobacteria

Methylobacterium

Unclassified Caulobacteraceae

Unclassi

Delftia

Unclassified Bacillales

Brevibacterium

Brachybacterium

Propionibacterium

Planomicrobium

Exiguobacterium

Unclassified Lactobacillales

Acinetobacter

Caulobacter

Stenotrophomonas

Unclassified Rhodobacteraceae

Salinicoccus

Planococcus

Staphylococcus

Corynebacterium

Unclassified Actinomycetales

Unclassified Actinomycetales

Unclassified Entero

Bacteria unclassified

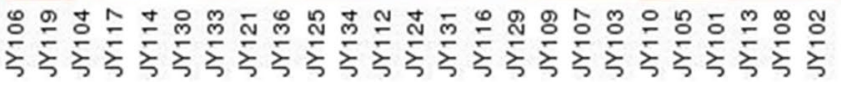

Figure 2. Heatmaps on bacterial abundance in twenty-five yeast strains at; (a), phylum level; (b), genus level. Other details as in Fig. 1. OTU data was first filtered and normalised using total sum-scaling in MicrobiomeAnalyst web-based tool ${ }^{27}$. The heatmaps were then constructed using Heatmapper web-based tool ${ }^{29}$. Clustering was applied to both column and row data with average linkage method and distances were measured as Euclidean distances. Red colour indicates high abundance and blue colour indicates low abundance as given in the colour scale on the top of the maps.

Bacterial community of Pichia spp. Bacterial reads of $P$. kudriavzevii (JY105, JY112, JY116, JY129 and JY131) and P. kluyveri (JY136) were compared similarly at bacterial phylum and genus level. Strain JY131 had maximum number of OTUs of 5524 and strain JY105 had minimum number of 1015. Diversity measures for Pichia spp. (Fig. 4Ia,Ib) were calculated as done for Candida spp. Firmicutes constituted $46 \%$ and was the most 


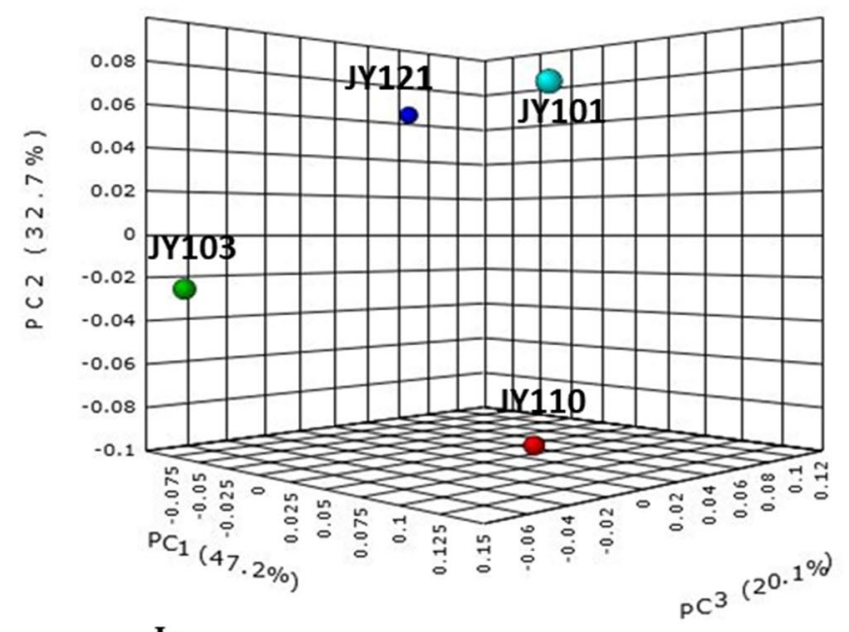

Ia

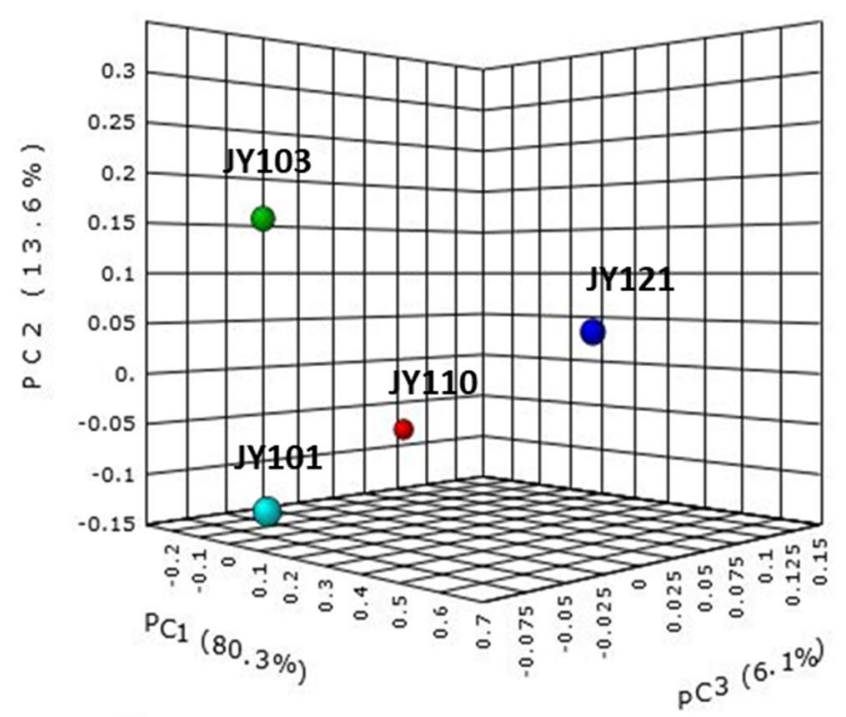

IIa
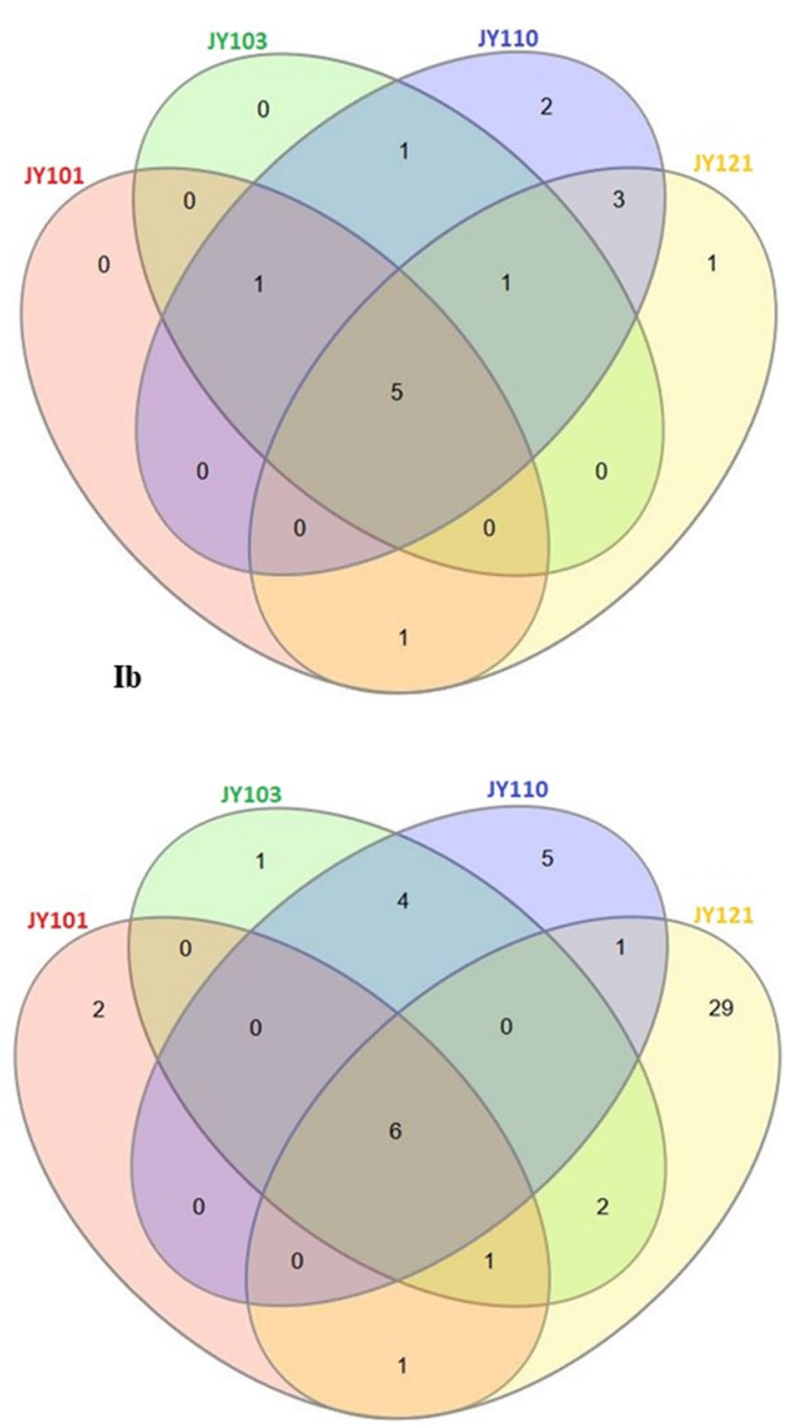

IIb

Figure 3. Bacterial diversity among different Candida spp. for intra-genera comparison of yeast strains; (Ia, Ib) PCoA plot and Venn diagram of bacterial phyla distribution in yeast, (IIa, IIb) PCoA plot and Venn diagram of bacterial genera distribution in yeast. principal coordinates analysis (PCoA) plots were constructed at both phylum and genus level using MicrobiomeAnalyst web-based tool ${ }^{27}$ for the assessment of bacterial beta-diversity between Candida strains using Bray-Curtis index. Venn diagram constructed using InteractiVenn web-based tool $^{28}$ to compare common and unique bacterial phyla and genera of the Candida strains. Other details as in Fig. 1. Summary of common and unique bacterial taxa derived from Venn diagram is explained in Tables S1 and S2.

abundant of the reads among all Pichia strains. Acidobacteria $(\sim 0.3 \%)$, Actinobacteria $(\sim 23 \%)$, Bacteroidetes $(\sim 2 \%)$, Firmicutes $(\sim 46 \%)$, Planctomycetes $(\sim 0.2 \%)$, Proteobacteria $(\sim 25 \%)$ and unclassified bacteria $(\sim 3 \%)$ were the common phyla for all strains (Fig. 3Ia; Table S5). Whereas Chlorobi and Deinococcus-Thermus were found to be specific for strain JY129 (Fig. 4IIa,IIb; Table S5).

At genus level, Staphylococcus ( 8\%), Propionibacterium ( 3\%), Streptococcus ( 2\%) and Corynebacterium $(\sim 1 \%)$ OTUs were common in the total read share. Genus Planococcus was present in all strains except JY105, showing high read dominance and contributing a total of $13 \%$ of OTU count (Fig. 4IIa,IIb). Reads of Weissella were only found in yeast strain JY136. Reads of unclassified Actinomycetales, Granulicatella, Gemella, unclassified Lactobacillales and unclassified Pseudomonadaceae were present only in yeast strain JY105 (Table S6). Unclassified Micromonosporaceae OTUs were uniquely present in strain JY129. Yeast strain JY131 had the OTUs of genus Siphonobacter. Further diversity analysis of different strains of the genus Pichia are given in Table S6 and Venn diagram (Fig. 4IIb).

Diversity indices of all yeast strains. Comparative metagenome diversity of all the 25 yeasts showed bacterial read heterogeneity among all the yeast as analysed by diversity indices (Fig. 5), bar plots (Fig. 1a,b) and 

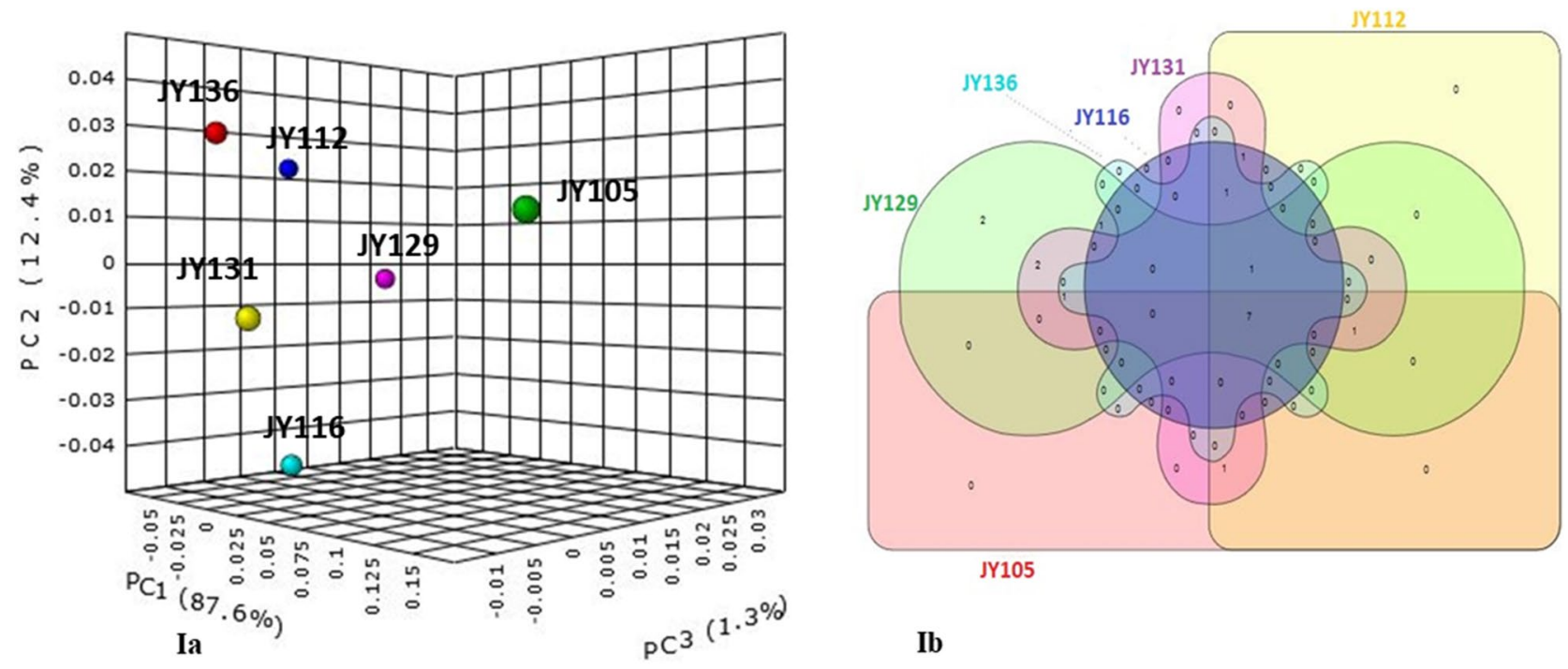

Ib
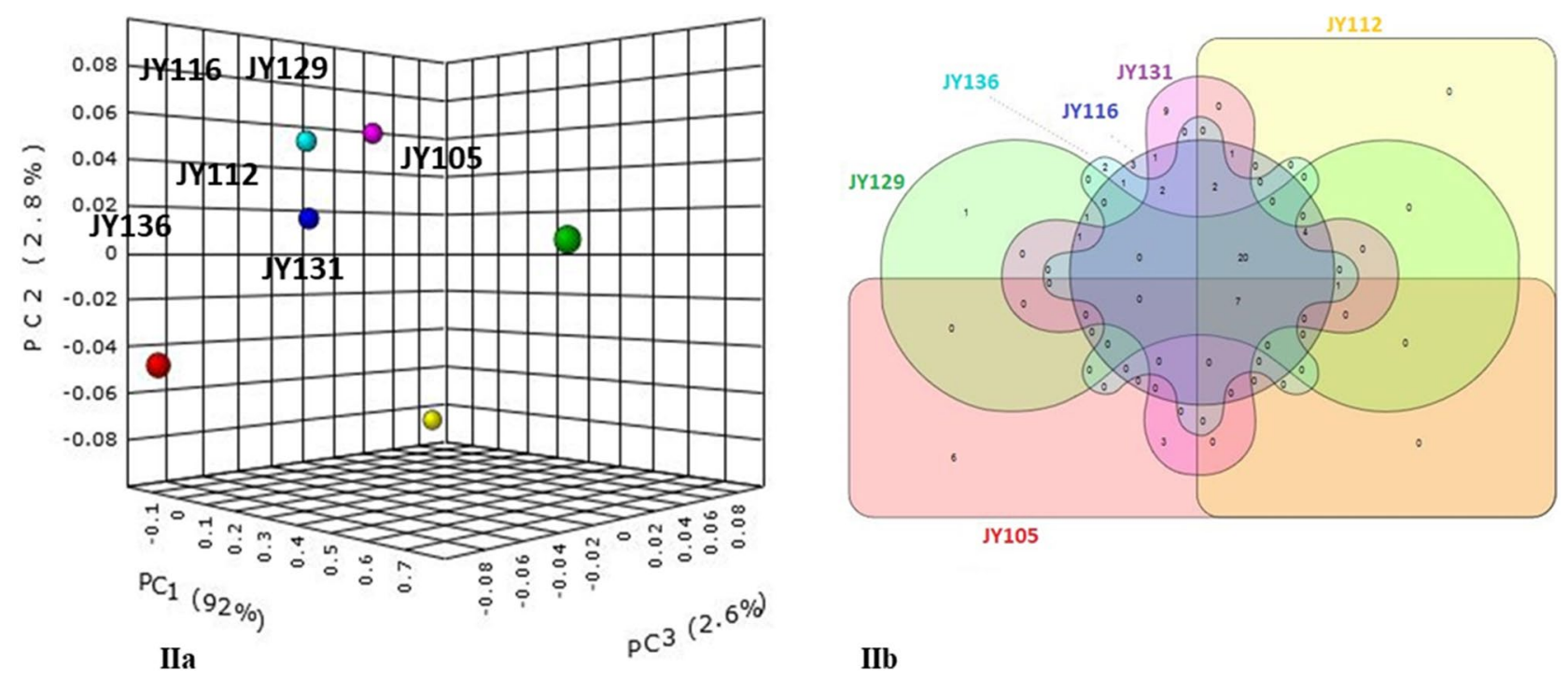

Figure 4. Bacterial diversity among different Pichia sp for intra-genera comparison of yeast strains; (Ia, Ib) PCoA plot and Venn diagram of bacterial phyla distribution in yeast, (IIa, IIb) PCoA plot and Venn diagram of bacterial genera distribution in yeast. Other details in Fig. 3. Summary of common and unique bacterial taxa derived from Venn diagram is explained in Tables S3 and S4.

heatmaps (Fig. 2a,b). The maximum number (5539) of OTUs was seen for $P$. kudriavzevii JY131 whereas the least (218 reads) was found in C. tropicalis JY106. Alpha diversity using the Chao-1 index and T-test/ANOVA statistical method (Fig. 5Ia,IIa) and beta diversity using Bray-Curtis index and PERMANOVA statistical method were examined (Fig. 5Ib,IIb) to validate our diversity analysis. Several unclassified reads were also detected at various taxonomic levels, with unclassified bacterial reads at phylum level contributing to significant abundance in strains JY106 (27\%), JY109 (13\%), JY110 (18\%) and JY119 (33\%) which would either need further analysis or corroboration.

Firmicutes $(\sim 45 \%)$ remain the dominant phylum followed by Proteobacteria $(\sim 25 \%)$ and Actinobacteria $(\sim 22 \%)$ for all the yeasts examined in this study (Figs. 1a, 2a). At the genus level, major differences in the abundance were observed (Figs. 1b, 2b). Only a few bacterial genera were common among certain groups of yeast strains, as shown by the clustering of these samples in the PCoA plots (Fig. 5IIb). Reads of the genus Streptococcus contributed the greatest number of OTUs in all yeast strains, with the maximum number in C. metapsilosis strain JY103 ( 34\% of their total OTUs) and the minimum in C. suratensis strain JY121 ( 4\% of their total). Strain JY119 was the only strain that did not possess any Streptococcus reads. It had notably different read composition from the rest of the yeast strains where Acidobacteria (236 OTUs) was the most dominant phylum. Also, JY119 showed lesser richness of bacterial diversity as against the majority of the yeast strains. Planococcus reads dominated in strains JY112, JY116, JY121, JY124, JY125, JY129, JY130, JY131, JY133, JY134 and JY136 with the maximum number of OTUs in Candida suratensis strain JY124 ( $20 \%$ of their total) and the minimum in P. kudriavzevii strain JY131 ( 11\% of their total). OTUs of unclassified Clostridiales were only observed in $P$. 


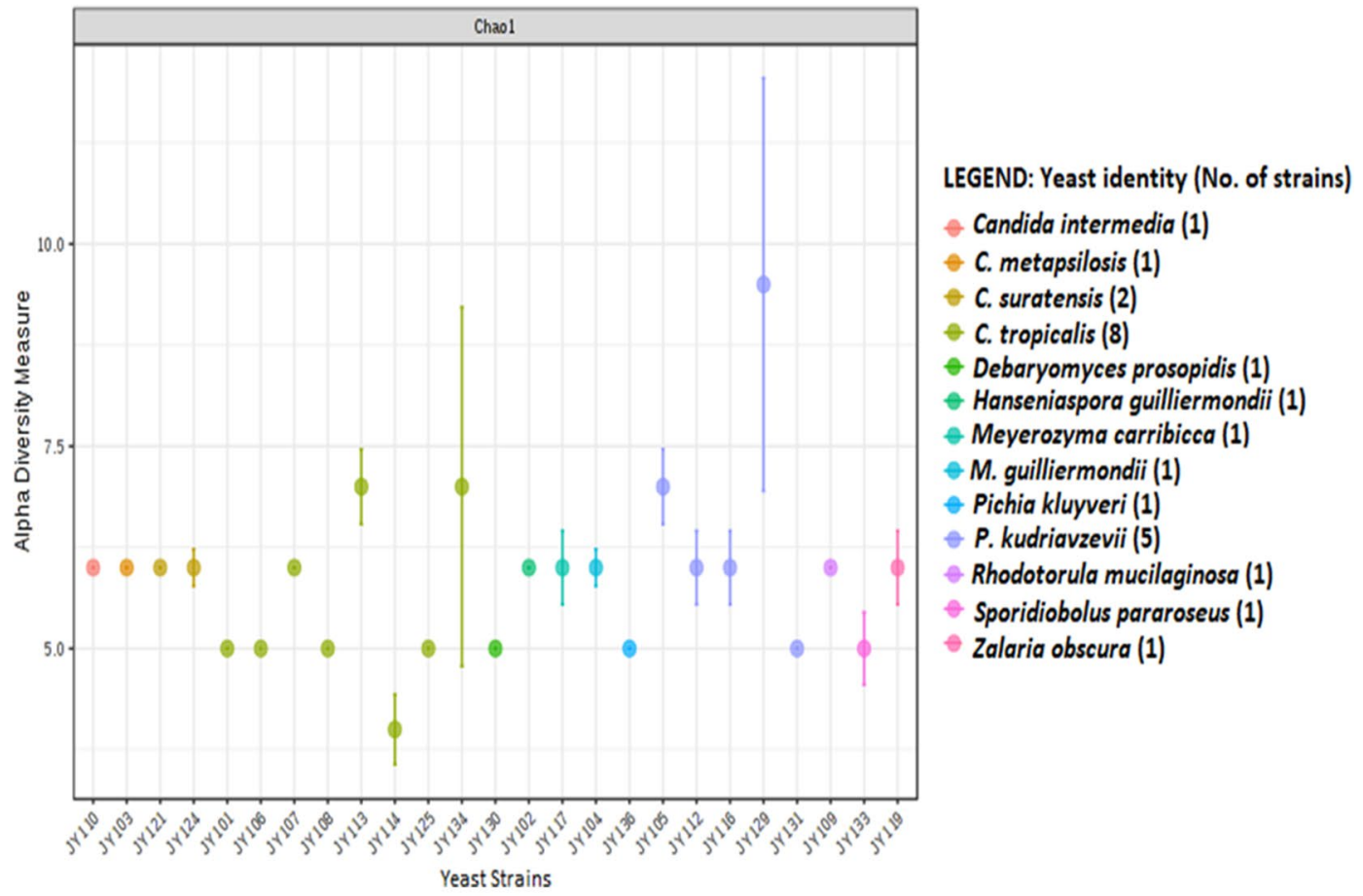

Ia

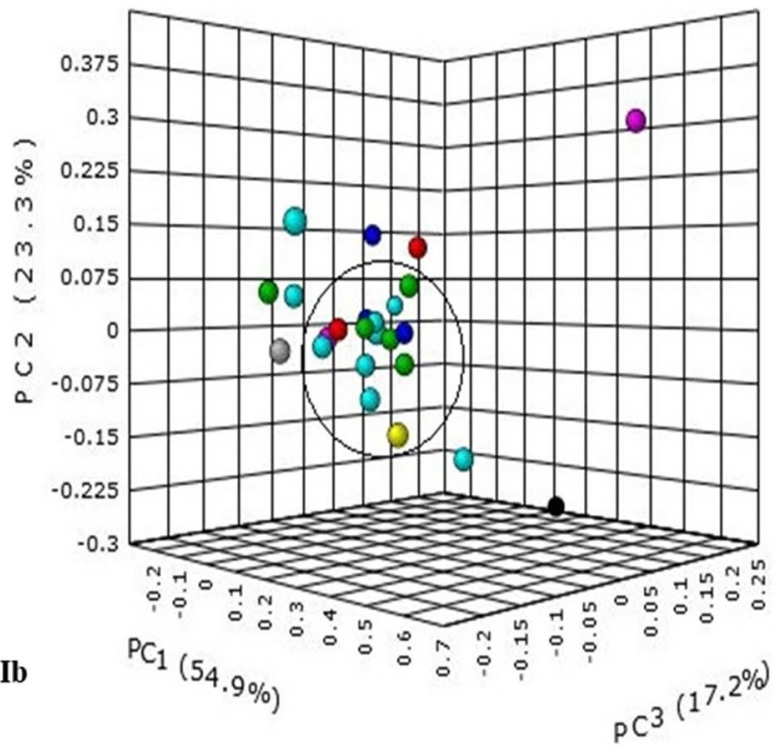

LEGEND: Yeast identity (No. of strains)

- Candida tropicalis (8)

O Hanseniaspora guilliermondii (1)

- C. metapsilosis (1)

- Meyerozyma guilliermondii (1)

- Pichia kudriavzevii (5)

- Rhodotorula mucilaginosa (1)

- C. intermedia (1)

O M. carribicca (1)

- Zalaria obscura (1)

- C. suratensis (2)

- Debaryomyces prosopidis (1)

- Sporidiobolus pararoseus (1)

- P. kluyveri (1)

Figure 5. Bacterial diversity among all twenty-five yeast strains; (Ia, Ib) Alpha and beta diversity of bacterial phyla distribution in all yeast, (IIa, IIb) Alpha and beta diversity of bacterial genera distribution in all yeast. Alpha diversity was measured by Chao-1 index and beta diversity with PCoA plots was measured with BrayCurtis index at bacterial genus and phylum levels for all yeast strains. Other details as in Figs. 3 and 4. Summary of common and unique bacterial taxa derived from Venn diagram is explained in Tables S5 and S6.

kudriavzevii JY131. Diversity and abundance of the bacterial community of the yeasts have been represented in the form of bar graph and heat map at phylum as well as at genus level (Figs. 1a,b, 2a,b).

Insights into bacteria associated with Candida tropicalis strain JY101. We selected C. tropicalis strain JY101 as a model yeast for further studies to validate the bacterial diversity. This was done through FISH studies followed by cultivating the bacteria from the yeast and re-infecting the yeast strain using a fluorescently labelled bacterium to mimic Koch's postulates. 

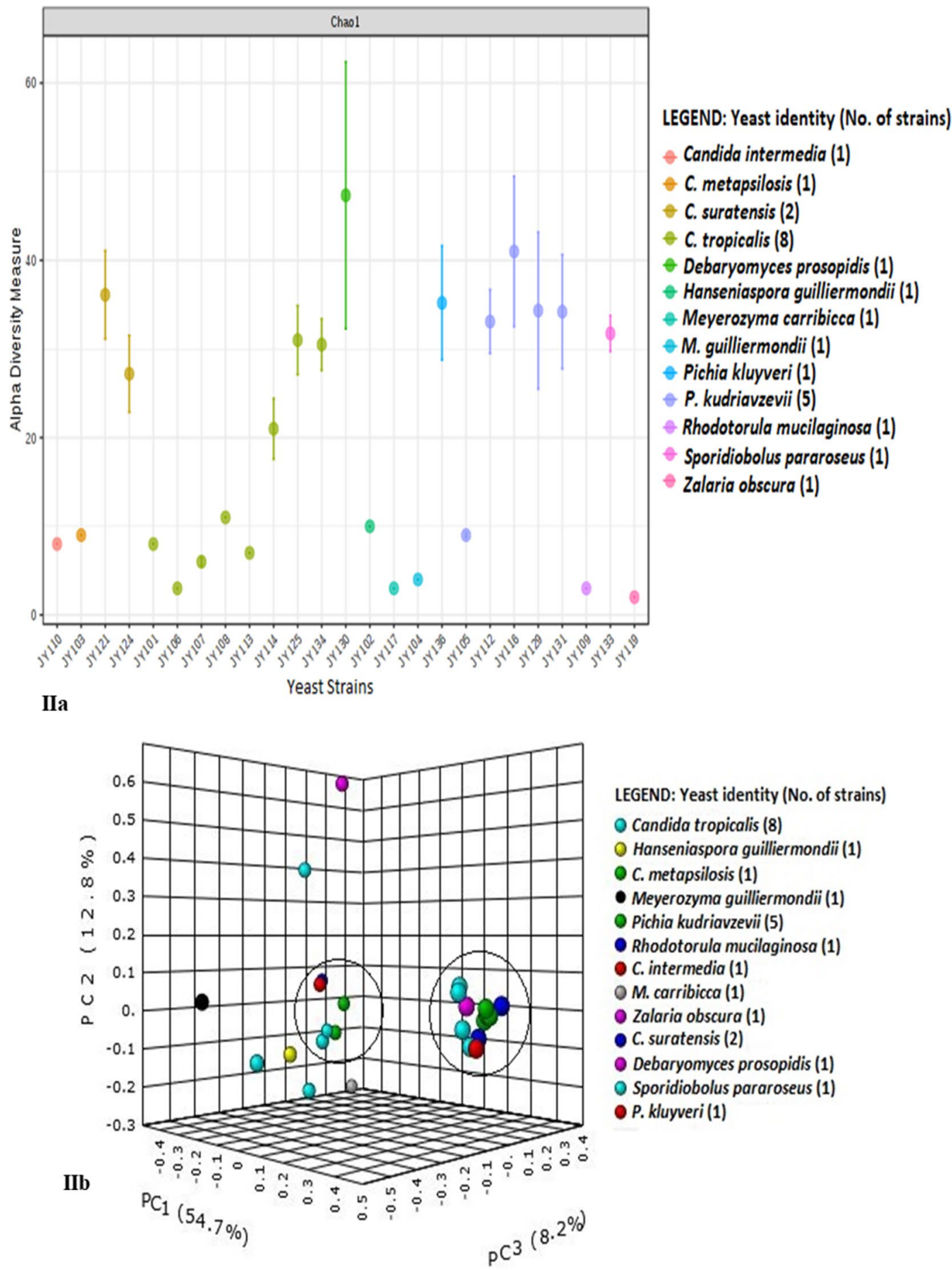

Figure 5. (continued)

FISH studies. To corroborate the presence of bacterial communities in yeast, FISH was performed with $C$. tropicalis using Cy3 labelled bacterial specific probe, EUB338. Positive fluorescence observed (Fig. 6a) confirms the presence of endo-bacteria of $C$. tropicalis. We rule out the possibility of positive fluorescence by yeast mitochondria since isolated mitochondria did not show any positive fluorescence using the above probe (data not shown).

Cultivated and characterized bacteria from yeast. $\quad 16 \mathrm{~S}$ rRNA gene metagenome of C. tropicalis JY101 showed reads of unclassified Pseudomonadaceae, members of which can be cultured easily. Cells of strain JY101 were ruptured with physical (glass beads) and enzymatic (zymolase) treatments. When plated on nutrient broth, this yielded four different bacteria which were purified on nutrient agar (HIMEDIA, M002) and sequenced for $16 \mathrm{~S}$ rRNA gene-based identification. The bacteria identified were members of genus Pseudomonas, Chryseo- 


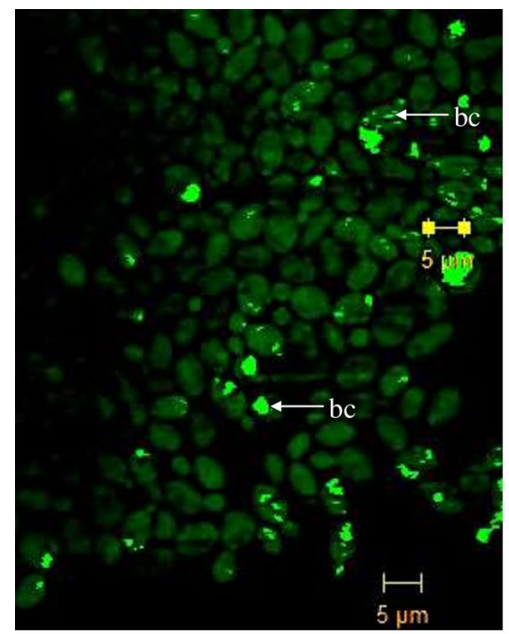

a

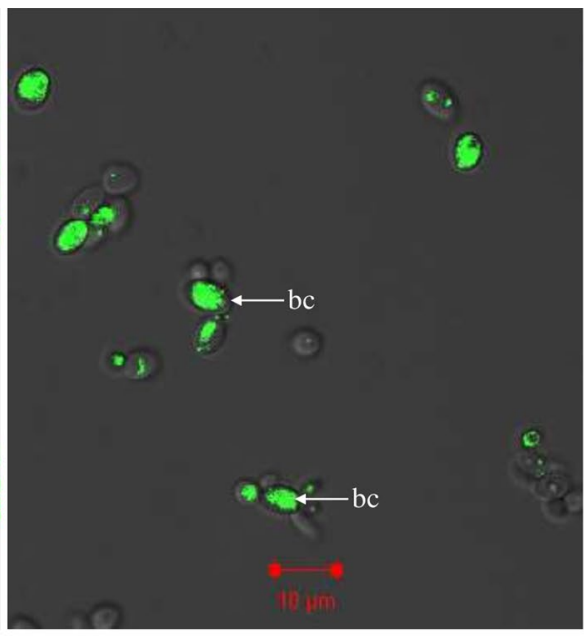

b

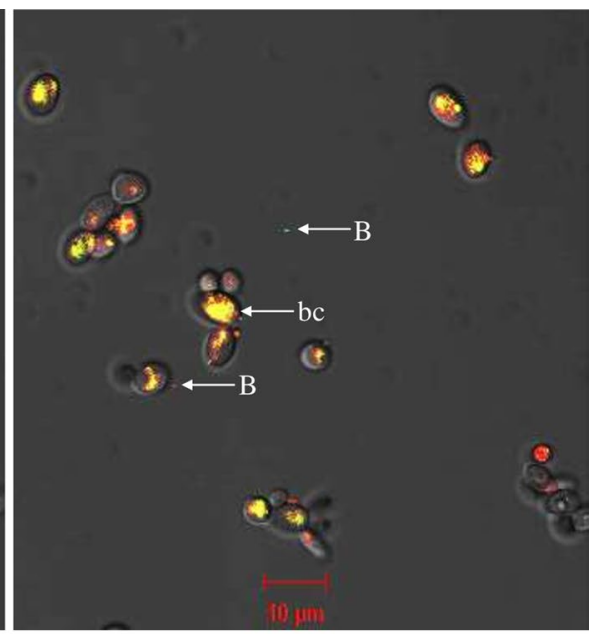

C

Figure 6. Confocal fluorescence microscopic FISH images of Candida tropicalis. (a) Native bacterial community (bc) of the yeast showing the green fluorescence inside the yeast cells (b) Infection studies showing native bacterial community (bc) of the yeast showing green fluorescent while the mCherry tagged P. stutzeri have red fluorescent and an over lay of the image (c) shows the presence of both native community along with the infected cells inside the yeast cells. Bacterial communities associated with the yeast cells were stained with rRNA bacterial EUB338-Cy3 probe (green). Arrows indicate: bc, bacterial communities; B, Bacterial cells which are seen outside the yeast.

bacterium, Lysinibacillus and Propionibacterium. EzTaxon BLAST search of the 16S rRNA gene sequence (1387 nt) of Pseudomonas strain JC703 had the highest (99.9\%) identity with Pseudomonas stutzeri ATCC $17588^{\mathrm{T}}$ followed by other members of the genus Pseudomonas with $<99 \%$ sequence identity. While the Chryseobacterium strain JC507 sequence (1428 nt) had highest (98.7\%) identity with Chryseobacterium indologenes NBRC $14944^{\mathrm{T}}$ followed by other members of the genus Chryseobacterium with $<98.6 \%$ identity. Lysinibacillus strain JC1018 sequence (889 nt) had the highest (100\%) identity with Lysinibacillus fusiformis NBRC $15717^{\mathrm{T}}$ followed by $<98.7 \%$ with other members. Propionibacterium strain JC704 sequence (1396 nt) had the highest similarity (98.6\%) with Propionibacterium acne DSM $1897^{\mathrm{T}}$ followed by $<95.7 \%$ with other members. The $16 \mathrm{~S}$ rRNA gene sequences of the four bacteria of $C$. tropicalis JY101 were deposited with NCBI with accession numbers LR735276, LT838865, LR735277 and LR743671 respectively for bacterial strains JC703, JC507, JC1018 and JC704. All the cultures have been preserved as glycerol (50\% v/v) stocks stored at $-20^{\circ} \mathrm{C}$ and Chryseobacterium sp. JC507 was deposited with KCTC (KCTC 52928) and NBRC (NBRC 113872) as Chryseobacterium candidae JC507 ${ }^{\mathrm{T}}$. Our attempts failed to cultivate other bacteria from C. tropicalis JY101 where we used Marine agar media (HIMEDIA, M384), Mueller Hinton agar (HIMEDIA, M173) and Thiobacillus agar media (HIMEDIA, M788).

Infection studies of C. tropicalis JY101. For infection studies, we used auto-fluorescent bacteria Prochlorococcus sp. and Rhodopseudomonas palustris TIE-1 and mCherry labelled plasmid transformed in $P$. stutzeri JC703, E. coli DH5a and S. typhimurium. The mCherry tagged E. coli DH5a or Salmonella did not infect C. tropicalis JY101 nor were observed in the yeast whereas the other bacteria yielded successful infection and entry into the yeast in nitrogen deficient media. In C. tropicalis JY101, Prochlorococcus sp. was observed as green fluorescent bacteria (Fig. 7a), Rhodopseudomonas palustris TIE-1 cells were seen as red fluorescent bacteria (Fig. 7b) and mCherry labelled P. stutzeri JC703 was observed as red fluorescent bacteria (Fig. 7c,d). The control yeast cells exhibited spore like structures (Fig. 7e) that were not seen in the case of infected yeast cells. Scanning Electron Microscopy (SEM) of infected C. tropicalis with P. stutzeri helped to further observe the yeast and bacteria (Fig. 8). Bacteria were first seen to adhere to the yeast cell in many numbers (Fig. 8a; counter supported by confocal image Fig. 8a1); particularly at the collar of the budding yeast (Fig. 8b; counter supported by confocal image Fig. 8b1) and were seen inside the yeast cell (Fig. 7d,e; Movie S3). FISH analysis of the C. tropicalis along with the infected mCherry tagged $P$. stutzeri showed positive signals on hybridization. Along with the native bacterial community of the yeast (Fig. 6b) which imparts green fluorescence when bind with bacterial rRNA probe EUB338-Cy3 (Fig. 6b), red fluorescence was also observed which was due to mcherry tagged P. stutzeri. An overlay image (Fig. 6c) confirms the presence of inherent bacterial community and the infected cells of $P$. stutzeri inside the yeast (Fig. S4).

\section{Discussion}

Endosymbiotic bacteria play an important role in the evolution of higher life forms ${ }^{35-37}$. In this process, some of the endosymbiotic bacteria became integral components of the host as organelles, while a few remained as endosymbionts of fungi $i^{38}$, plants ${ }^{39}$ and animals ${ }^{40}$. Endosymbionts have been extensively studied from an evolutionary point of view ${ }^{41}$. To this end, recent studies also tried to establish the endosymbiotic theory of evolution of mitochondria using engineered E. coli into Saccharomyces cerevisiae ${ }^{20,21}$. While these are artificially generated 


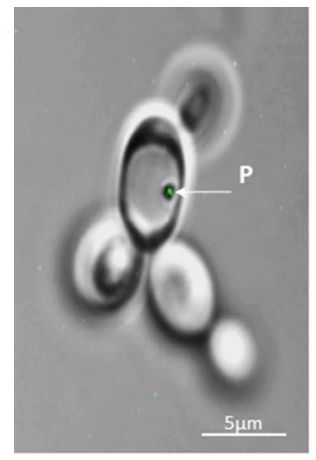

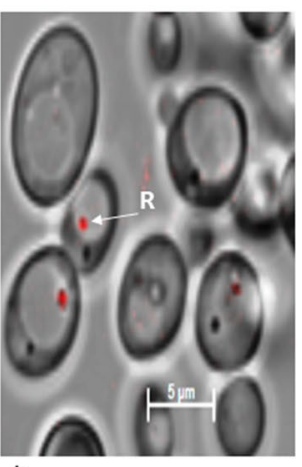

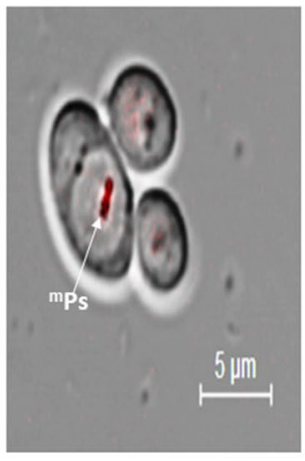

c

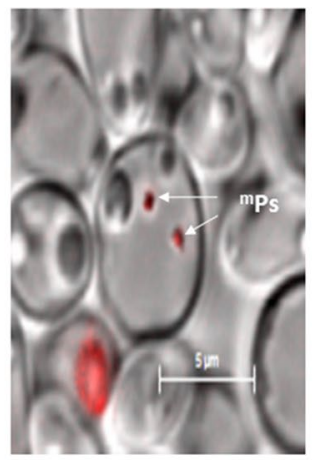

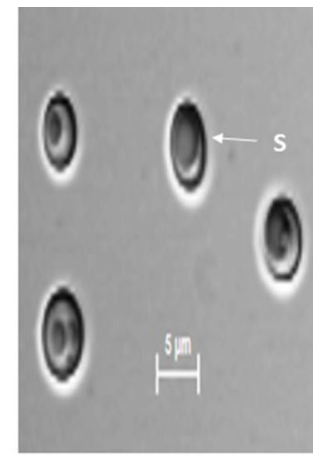

Figure 7. Confocal microscopy images of yeast-bacteria co-cultures under nitrogen deficit showing (a) Prochlorococcus sp. as green fluorescent bacteria in C. tropicalis, (b) Rhodopseudomonas palustris strain TIE-1 as red fluorescent bacteria in C. tropicalis, (c,d) mCherry tagged P. stutzeri as red fluorescent bacteria in C. tropicalis, (e) Control C. tropicalis cells grown in nitrogen deficiency without bacteria. Images were magnified with total magnification of $630 \mathrm{X}$ with appropriate scale. Arrows indicate: P, Prochlorococcus; $\mathrm{R}$, Rhodopseudomonas; ${ }^{\mathrm{m} P s,}$ mCherry Pseudomonas; S, Spore.

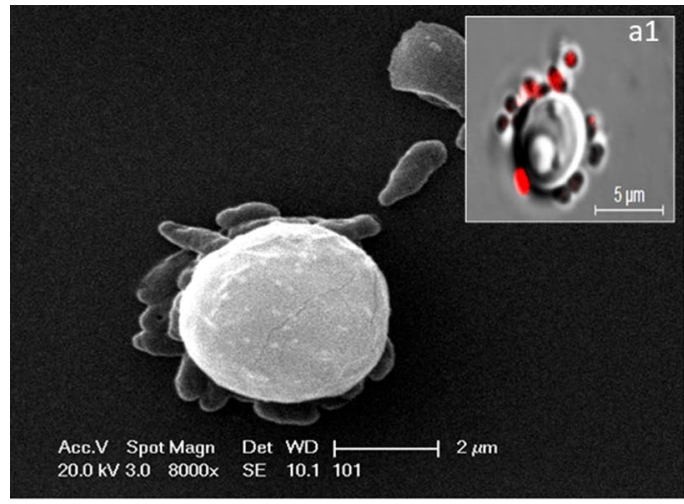

a

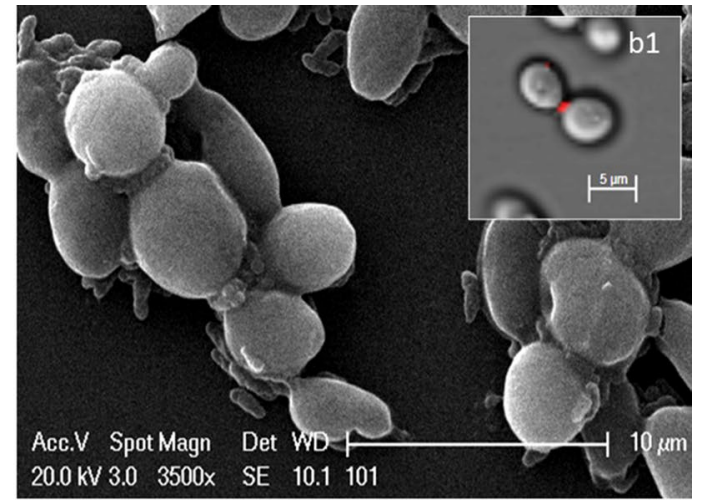

b

Figure 8. Scanning electron microscopy (SEM) images showing (a) P. stutzeri bacteria attachments on a $C$. tropicalis cells, (b) P. stutzeri attachments onto C. tropicalis bud collars. Insert: (a1) Confocal microscope image showing similar $P$. stutzeri attachment on a C. tropicalis cell, (b1) Confocal microscope image showing similar $P$. stutzeri attachment onto budding C. tropicalis cells.

endosymbionts, naturally harboured "endobacteria" of yeast have only now come under investigation ${ }^{12-19}$, implicating these bacteria in a variety of roles. Our study aimed to extend the understanding of co-occurrence of yeast and bacteria, by first examining the diversity through $16 \mathrm{~S}$ rRNA gene metagenome analysis and then with infection assays of yeast with fluorescent bacteria.

Yeast cultures were comprehensively subjected to various methods to ensure absence of external bacteria or any other contamination. Initially, BLBs moving inside the yeast were examined for viability using ViaGram staining kit that determines both viability and bacterial nature. This showed that the bodies are viable and could potentially have bacterial origin (Fig. S1). We performed FISH, which is a powerful tool to detect bacteria directly, without the need for cultivation. The rRNA bacterial probe EUB338-Cy3 has been routinely employed and demonstrated to have sufficient power to detect most bacteria. FISH analysis with this probe revealed successful hybridization of the bacteria inside the cells of $C$. tropicalis (Fig. 6a). Repeated streaking and/or subculturing of yeast strains on multiple growth media did not yield any visible bacterial colonies (data not shown). Taken together, these evidences provide a strong case for the hypothesis that these BLBs are indeed bacteria associated within the yeast. With these leads, we analysed the bacterial communities with both culture independent and dependent techniques.

The 16S rRNA gene metagenome analysis showed that about $85 \%$ of the yeasts examined in possess bacterial reads and also the presence of the rich diversity of bacteria associated with yeast. Isolation of yeast-like fungus, i.e., Zalaria obscura JY119 presented us with an opportunity to utilize its system as an out-group in our study of yeasts. It was intriguing to note that the bacterial diversity of JY119 was markedly different from the rest of the yeast as can be seen from the bar plots and heatmaps. This evidently conveys the specificity of bacterial diversity among fungal organisms. We analysed yeast genera-specific diversity patterns (Figs. 1b, 2b, 5IIa,IIb) 
and those spanning among all the strains (Table 1). Overall, taxa of the phylum Firmicutes dominated in all the yeasts analysed in this study. This may be attributed to their ubiquitous nature and the existence of its numerous spherical cell shaped members because the dominant Firmicutes taxa of yeasts were Streptococcus, Staphylococcus and Planococcus (Figs. 1,2). In correlation with these data, the moving BLBs first seen in yeast cells were also coccoidal. This could point to the significance of the shape of the bacteria present inside yeast cells. Also, several reads of rod-shaped bacteria notably from unclassified Pseudomonadaceae family were obtained. Possible heterogeneity in the morphology of these bacteria cannot simply be explained (in this case) through phase contrast microscopic studies (Fig. S1a) or from FISH studies (Fig. 6a). However, the morphological distinction in cell shape and size can be clearly seen in Movie S2 where, if closely observed, two distinct morphological structures i.e., one stained with green is distinct from another BLB stained red.

Often metagenome results on microbiota are linked to the properties of the habitat from where DNA is isolated. However, from our results, the bacterial diversity did not show any dependence on environmental conditions from which the yeast hosts were isolated (Fig. 1). Instead, the diversity was specific to the yeast host itself, as indicated by the comparison among Candida and Pichia species (Figs. 3, 4, S3). However, without more experimentation, these correlations are only at the best, suggestive. This culture-independent method enabled us to explore the existence of a bacterial "community" as against studies reporting the presence of single bacterial species inside the yeast cell ${ }^{12-17}$. Also, as many as $10^{7}$ live bacteria were counted in a single spore of the Arbuscular Mycorrhizal Fungus, Gigaspora margarita ${ }^{42}$ which supports the existence of communities. Uncovering a wealth of bacterial reads from twenty-five different yeasts in this study reveals substantial numbers of bacteria that cannot be easily dismissed. Consequently, future studies should focus on understanding where and how the yeast hosts these bacteria.

Further, the data was validated to an extent by our successful attempts to isolate some of these bacteria from C. tropicalis JY101 by culture-dependent methods. Out of the four isolated bacteria, three (Pseudomonas stutzeri JC703, Lysinibacillus fusiformis JC1018, Chryseobacterium sp. JC507) were successfully sub-cultured and preserved, while Propionibacterium acnes JC704 (renamed as Cutibacterium acnes; Ref. ${ }^{43}$ ) lost its viability soon after its isolation. It has been demonstrated that many obligate fungal endosymbionts such as "Candidatus Glomeribacter gigasporarum" are uncultivable ${ }^{44}$. Perhaps, Propionibacterium acnes has become obligately associated with yeast maybe by the loss of some essential genes making it dependent on the host. The reads of Propionibacterium acnes were common to all the 25 yeast strains that again begs the question of its obligate relationship. Interestingly, $P$. acnes has been observed to adapt to new organisms as an endosymbiont, displaying flexibility for hosts ${ }^{45}$. Therefore, it may not be unusual to note the presence of Propionibacterium along with yeast cells. Among those that were sub-cultured, Chryseobacterium sp. JC507 was described as a new species and named as Chryseobacterium candidae ${ }^{13}$. Out of 12 genera identified through the metagenome of $C$. tropicalis, only members of three genera could be successfully cultivated in this study. Hence, a large number ( 95\%) remains yet-to-be cultivated or lost cultivability, indicating an unexplored black box of diverse microbiota of yeast.

The function and role of these bacteria of yeast are still to be elucidated, particularly concerning the host which bears the cost of harbouring them (Fig. 6a). The recent finding on interactions between the yeast Rhodotorula mucilaginosa harbouring $\mathrm{N}_{2}$-fixing $P$. stutzeri and rice plants highlights the role of endosymbiotic bacteria in the nitrogen needs of plants ${ }^{18}$. This example parallels the role of endobacteria of plant-associated filamentous fungi ${ }^{19}$. Similarly, certain species of the isolated bacteria from our study as well as Rhodopseudomonas palustris used for infection assays, are known to harbour nif genes that help in nitrogen fixation ${ }^{46,47}$. We hypothesize that this capability could be of use to the yeast host and potentially form a bipartite system. We started with a simple strategy to infect a yeast host with the isolated bacteria. Co-cultures of $C$. tropicalis JY101 along with fluorescently labelled $P$. stutzeri JC703 showed the establishment of $P$. stutzeri JC703 inside the yeast cell under nitrogen crisis. The control cultures of $C$. tropicalis JY101 without $P$. stutzeri formed spore like structures with poor growth (Fig. 7e). Devoid of a helpful partner, the control yeast cells possibly coped in the absence of an important nutrient like nitrogen by forming spores. These observations support our hypothesis that $P$. stutzeri and other bacteria aid the host under nitrogen deprivation, providing scope for further analysis. Although no reads of Prochlorococcus sp. were observed in the yeast metagenome, we have seen its entry into the yeast under nutrition deficiency (Fig. 7a). We speculate that this demonstrates the ability of the yeast to acquire bacteria, if the situation demands. In such cases, these bacteria could be temporary residents where they may be considered as beneficial guests to the yeast under stress. This is unlike the case where bacteria like Rickettsia that have, through obligate endosymbiosis, evolved into organelles like mitochondria. These interesting findings of infection should be carefully explained with supporting experimental designs that can unravel the molecular events controlling this phenomenon and critically probe the process of bacterial infection of yeast. At this stage comparison of the massive diversity from metagenome with the infection of one or two bacteria of the yeast still raises a question on this anomaly.

Our study for the first time shows the existence of large bacteria communities in yeast. Collectively, present and future studies will provide insights into the ecology and evolution of the bond between a host and its endosymbiont. Genome size reduction of $E$. coli engineered as a yeast endosymbiont ${ }^{21}$ describes a crucial stage in the evolution of eukaryotic organelles. This type of event must be looked for in natural yeast-bacterial systems as well, though we argue that we cannot yet firmly categorize which of these bacteria are permanent or transient or whether they are indeed obligate endobacteria. Moreover, we realise that the current evidences from this and previous studies fall short in addressing the function of yeast associated bacteria as our work aimed to provide a preliminary examination into vexing conundrum of ghost bacterial communities of yeast. 


\section{Data availability}

Raw FastQ files of the 16S rRNA metagenomes of all 25 yeast strains were deposited in National Center for Biotechnology Information Sequence Read Archive and are available in NCBI SRA under accession numbers given in Table 1 .

Received: 5 October 2020; Accepted: 15 April 2021

Published online: 30 April 2021

\section{References}

1. Nowack, E. C. \& Melkonian, M. Endosymbiotic associations within protists. Philos. Trans. R. Soc. B Biol. Sci. 365, 699-712 (2010).

2. Pawlowska, T. E. et al. Biology of fungi and their bacterial endosymbionts. Annu. Rev. Phytopathol. 56, 289-309 (2018).

3. Deveau, A. et al. Bacterial-fungal interactions: ecology, mechanisms and challenges. FEMS Microbiol. Rev. 42, 335-352 (2018).

4. Frey-Klett, P. et al. Bacterial-fungal interactions: hyphens between agricultural, clinical, environmental, and food microbiologists. Microbiol. Mol. Biol. Rev. 75, 583-609 (2011).

5. Álvarez-Pérez, S., Lievens, B. \& Fukami, T. Yeast-bacterium interactions: the next frontier in nectar research. Trends Plant Sci. 24, 393-401 (2019).

6. Mondo, S. J. et al. Bacterial endosymbionts influence host sexuality and reveal reproductive genes of early divergent fungi. Nat. Commun. 8, 1843 (2017).

7. Bonfante, P. \& Desirò, A. Who lives in a fungus? The diversity, origins and functions of fungal endobacteria living in Mucoromycota. ISME J. 11, 1727-1735 (2017).

8. Starmer, W. T. \& Lachance, M. A. Yeast ecology. In The Yeasts 5th edn (eds Kurtzman, C. et al.) 65-83 (Elsevier, 2011).

9. Lanhace, M. A. \& Pang, W. M. Predacious yeasts. Yeast 13, 225-232 (1997).

10. Reggiori, F. \& Klionsky, D. J. Autophagic processes in yeast: mechanism, machinery and regulation. Genetics 194, 341-361 (2013).

11. Belda, I., Zarraonaindia, I., Perisin, M., Palacios, A. \& Acedo, A. From vineyard soil to wine fermentation: microbiome approximations to explain the "terroir" concept. Front. Microbiol. 8, 821 (2017).

12. Salmanian, A. H. et al. Foodborne yeasts serve as reservoirs of Helicobacter pylori. J. Food Saf. 32, 152-160 (2012).

13. Indu, B. et al. Chryseobacterium candidae sp. nov., isolated from a yeast (Candida tropicalis). Int. J. Syst. Evol. Microbiol. https:// doi.org/10.1099/ijsem.0.003716 (2020).

14. Saniee, P. \& Siavoshi, F. Endocytic uptake of FITC-labelled anti-H. pylori egg yolk immunoglobulin Y in Candida yeast for detection of intracellular H. pylori. Front. Microbiol. 6, 113 (2015).

15. Tavakolian, A. et al. Localization of Staphylococcus inside the vacuole of Candida albicans by immunodetection and FISH. Inf. Genet. Evol. 75, 1-6 (2019).

16. Uribe-Alvarez, C. et al. Wolbachia pipientis grows in Saccharomyces cerevisiae evoking early death of the host and deregulation of mitochondrial metabolism. Microbiol. Open 8, e00675 (2019).

17. Kang, S. W., Jeon, B. Y., Hwang, T. S. \& Park, D. H. Symbiotic relationship between Microbacterium sp. SK0812 and Candida tropicalis SK090404. J. Microbiol. 47, 721-727 (2009).

18. Sen, D. et al. A unique life-strategy of an endophytic yeast Rhodotorula mucilaginosa JGTA-S1-a comparative genomics viewpoint. DNA Res. 26, 131-146 (2019).

19. Paul, K. et al. A tripartite interaction among the Basidiomycete Rhodotorula mucilaginosa, $\mathrm{N}_{2}$-fixing endobacteria and rice improves plant nitrogen nutrition. Plant Cell https://doi.org/10.1105/tpc.19.00385 (2019).

20. Mehta, A. P. et al. Engineering yeast endosymbionts as a step toward the evolution of mitochondria. Proc. Natl. Acad. Sci. U. S. A. 115, 11796-11801 (2018).

21. Mehta, A. P. et al. Toward a synthetic yeast endosymbiont with a minimal genome. J. Am. Chem. Soc. 141, 3799-13802 (2019).

22. Hoffman, C. S. Preparation of yeast DNA. Curr. Protoc. Mol. Biol. 39, 13-11 (1997).

23. Weiss, S., Samson, F., Navarro, D. \& Casaregola, S. YeastIP: a database for identification and phylogeny of ascomycetous yeasts. FEMS Yeast Res. 13, 117-125 (2013).

24. Schloss, P. D. et al. Introducing mothur: open-source, platform-independent, community-supported software for describing and comparing microbial communities. Appl. Environ. Microbiol. 75, 7537-7541 (2009).

25. Schloss, P. D., Gevers, D. \& Westcott, S. L. Reducing the effects of PCR amplification and sequencing artifacts on $16 \mathrm{~S}$ rRNA-based studies. PLoS ONE 6, e27310 (2011).

26. Pruesse, E. et al. SILVA: a comprehensive online resource for quality checked and aligned ribosomal RNA sequence data compatible with ARB. Nucl. Acids Res. 35, 7188-7196 (2007).

27. Dhariwal, A. et al. Microbiome analyst: a web-based tool for comprehensive statistical, visual and meta-analysis of microbiome data. Nucl. Acids Res. 45, 180-188 (2017).

28. Heberle, H., Meirelles, G. V., da Silva, F. R., Telles, G. P. \& Minghim, R. InteractiVenn: a web-based tool for the analysis of sets through Venn diagrams. BMC Bioinform. 16, 169 (2015).

29. Babicki, S. et al. Heatmapper: web-enabled heat mapping for all. Nucl. Acids Res. 44, 147-153 (2016).

30. Hammer, Ø., Harper, D. A. T. \& Ryan, P. D. PAST: Paleontological statistics software package for education and data analysis. Palaeontol. Electron. 4, 9 (2001).

31. Lakshmi, K. V. N. S., Sasikala, Ch., Takaichi, S. \& Ramana, Ch. V. Phaeospirillum oryzae sp. nov., a spheroplast forming phototrophic alphaproteobacterium from a paddy soil. Int. J. Syst. Evol. Microbiol. 61, 1656-1661 (2011).

32. Parsley, L. C., Newman, M. M. \& Liles, M. R. Fluorescence in situ hybridization of bacterial cell suspensions. Cold Spring Harbor Protoc. 2010, pdb-rot5493 (2010).

33. Lagendijk, E. L., Validov, S., Lamers, G. E., De Weert, S. \& Bloemberg, G. V. Genetic tools for tagging Gram-negative bacteria with mCherry for visualization in vitro and in natural habitats, biofilm and pathogenicity studies. FEMS Microbiol. Lett. 305, 81-90 (2010).

34. Zhao, F., Zhang, Y., Li, H., Shi, R. J. \& Han, S. Q. $\mathrm{CaCl}_{2}$-heat shock preparation of competent cells of three Pseudomonas strains and related transformation conditions. Chin. J. Appl. Ecol. 24, 788-794 (2013).

35. Deepshika, G., Mujahid, M., Lakshmi Prasuna, M., Sasikala, Ch. \& Ramana, Ch. V. iTRAQ-based quantitative proteomics reveals insights into metabolic and molecular responses of glucose-grown cells of Rubrivivax benzoatilyticus JA2. J. Prot. 194, 49-49 (2019).

36. Aanen, D. K. \& Eggleton, P. Symbiogenesis: beyond the endosymbiosis theory?. J. Theor. Biol. 434, 99-103 (2017).

37. Archibald, J. M. Endosymbiosis and eukaryotic cell evolution. Curr. Biol. 25, 911-921 (2015).

38. Bianciotto, V., Lumini, E., Bonfante, P. \& Vandamme, P. ‘Candidatus Glomeribacter gigasporarum' gen. nov., sp. nov., an endosymbiont of arbuscular mycorrhizal fungi. Int. J. Syst. Evol. Microbiol. 53, 121-124 (2003).

39. Santoyo, G., Moreno-Hagelsieb, G., del Carmen, O.-M. \& Glick, B. R. Plant growth-promoting bacterial endophytes. Microbiol. Res. 183, 92-99 (2016).

40. Perotti, M. A. et al. Rickettsia as obligate and mycetomic bacteria. FASEB J. 20, 2372-2374 (2006).

41. O’Malley, M. A. Endosymbiosis and its implications for evolutionary theory. Proc. Natl. Acad. Sci. U. S. A. 112, 10270-10277 (2015). 
42. Bianciotto, V. \& Bonfante, P. Arbuscular mycorrhizal fungi: a specialised niche for rhizospheric and endocellular bacteria. Anton. Van Leeuwenhoek 81, 365-371 (2002).

43. Dréno, B. et al. Cutibacterium acnes (Propionibacterium acnes) and acne vulgaris: a brief look at the latest updates. J. Eur. Acad. Dermatol. Venereol. 32, 5-14 (2018).

44. Salvioli, A., Lumini, E., Anca, I. A., Bianciotto, V. \& Bonfante, P. Simultaneous detection and quantification of the unculturable microbe 'Candidatus Glomeribacter gigasporarum' inside its fungal host Gigaspora margarita. New Phytol. 180, 248-257 (2008).

45. Campisano, A. et al. Interkingdom transfer of the acne-causing agent, Propionibacterium acnes, from human to grapevine. Mol. Biol. Evol. 31, 1059-1065 (2014).

46. Yan, Y. et al. Nitrogen fixation island and rhizosphere competence traits in the genome of root-associated Pseudomonas stutzeri A1501. Proc. Natl. Acad. Sci. 105, 7564-7569 (2008).

47. Cantera, J. J. L., Kawasaki, H. \& Seki, T. The nitrogen-fixing gene (nifH) of Rhodopseudomonas palustris: a case of lateral gene transfer?. Microbiology 150, 2237-2246 (2004).

\section{Acknowledgements}

IB and IS thank CSIR, New Delhi for the award for Senior and Junior research fellowship, respectively. Infrastructure facilities created through MHRH-IoE, DST-FIST, UGC-SAP (DRS) and TEQIP (for JNTU) are acknowledged. ChV thanks the DBT, for the award of TATA Innovation fellowship.

\section{Author contributions}

B.I., T.K. and S.I. collected the samples and performed the laboratory work. Bioinformatics work was performed by B.I., T.K. and U.J. and also wrote the first draft. C.V.R., C.S., B.I., T.K. and S.I. designed the experiments. C.V.R. and C.S. provided support for data analysis and writing the manuscript. All the authors have seen and approved the manuscript. There is no conflict of interest.

\section{Competing interests}

The authors declare no competing interests.

\section{Additional information}

Supplementary Information The online version contains supplementary material available at https://doi.org/ 10.1038/s41598-021-88658-x.

Correspondence and requests for materials should be addressed to C.V.R.

Reprints and permissions information is available at www.nature.com/reprints.

Publisher's note Springer Nature remains neutral with regard to jurisdictional claims in published maps and institutional affiliations.

(c) (i) Open Access This article is licensed under a Creative Commons Attribution 4.0 International License, which permits use, sharing, adaptation, distribution and reproduction in any medium or format, as long as you give appropriate credit to the original author(s) and the source, provide a link to the Creative Commons licence, and indicate if changes were made. The images or other third party material in this article are included in the article's Creative Commons licence, unless indicated otherwise in a credit line to the material. If material is not included in the article's Creative Commons licence and your intended use is not permitted by statutory regulation or exceeds the permitted use, you will need to obtain permission directly from the copyright holder. To view a copy of this licence, visit http://creativecommons.org/licenses/by/4.0/.

(C) The Author(s) 2021 